\title{
Development of fully Bayesian multiple-time-window source inversion
}

\section{AUTHOR(S):}

Kubo, Hisahiko; Asano, Kimiyuki; Iwata, Tomotaka; Aoi, Shin

\section{CITATION:}

Kubo, Hisahiko ...[et al]. Development of fully Bayesian multiple-time-window source inversion. Geophysical Journal International 2016, 204(3): 1601-1619

\section{ISSUE DATE:}

2016-01-28

URL:

http://hdl.handle.net/2433/226324

\section{RIGHT:}

This article has been accepted for publication in 'Geophysical Journal International' (c) The Authors 2016. Published by Oxford University Press on behalf of the Royal Astronomical Society. All rights reserved. 


\title{
Development of fully Bayesian multiple-time-window source inversion
}

\author{
Hisahiko Kubo, ${ }^{1, *}$ Kimiyuki Asano, ${ }^{2}$ Tomotaka Iwata ${ }^{2}$ and Shin Aoi $^{1}$ \\ ${ }^{1}$ National Research Institute for Earth Science and Disaster Prevention, Tsukuba, Ibaraki, Japan. E-mail: hkubo@bosai.go.jp \\ ${ }^{2}$ Disaster Prevention Research Institute, Kyoto University, Uji, Kyoto, Japan
}

Accepted 2015 December 16. Received 2015 December 15; in original form 2015 April 14

\begin{abstract}
S UMMAR Y
In the estimation of spatiotemporal slip models, kinematic source inversions using Akaike's Bayesian Information Criterion (ABIC) and the multiple-time-window method have often been used. However, there are cases in which conventional ABIC-based source inversions do not work well in the determination of hyperparameters when a non-negative slip constraint is used. In order to overcome this problem, a new source inversion method was developed in this study. The new method introduces a fully Bayesian method into the kinematic multiple-time-window source inversion. The multiple-time-window method is one common way of parametrizing a source time function and is highly flexible in terms of the shape of the source time function. The probability distributions of model parameters and hyperparameters can be directly obtained by using the Markov chain Monte Carlo method. These probability distributions are useful for simply evaluating the uniqueness and reliability of the derived model, which is another advantage of a fully Bayesian method. This newly developed source inversion method was applied to the 2011 Ibaraki-oki, Japan, earthquake $\left(M_{\mathrm{w}} 7.9\right)$ to demonstrate its usefulness. It was demonstrated that the problem with using the conventional ABIC-based source inversion method for hyperparameter determination appeared in the spatiotemporal source inversion of this event and that the newly developed source inversion could overcome this problem.
\end{abstract}

Key words: Inverse theory; Probability distributions; Earthquake source observations; Computational seismology.

\section{INTRODUCTION}

Earthquake source inversion is a geophysical inverse problem in which the spatial and/or temporal slip distribution of an earthquake is estimated from observed data (e.g. seismic waveform, geodetic and tsunami data). In most geophysical inverse problems, the classical least squares method is ineffective because the observed data are generally inaccurate and/or insufficient. One popular way of overcoming this challenge is to use a Bayesian inference approach and introduce prior information such as spatiotemporal smooth slips (e.g. Carlin \& Louis 2000; Tarantola 2005; Beck 2010). The basic formulation of the Bayesian inference approach is probabilistic (Duijndam 1988). This approach starts with the introduction of a prior probability distribution for model parameters, which plays the role of regularization. Given the observed data, this prior probability distribution is combined with a probability distribution of the observation equation using Bayes' theorem. This Bayesian approach is fundamental and applicable to all types of inverse problems. Source-inversion studies that use the Bayesian approach are roughly classified into two groups: those that use Akaike's Bayesian Information Criterion (ABIC; Akaike 1980) and those that use the fully Bayesian method.

ABIC was proposed by Akaike (1980) based on the entropy maximization principle. The first use of ABIC in earthquake source inversions was conducted by Yabuki \& Matsu'ura (1992). They formulated the source inversion with a smoothing slip constraint using the Bayesian inference formulation. Then, they used ABIC to objectively determine an optimal value for the relative weight of the smoothing constraint. This relative weight is often called the 'hyperparameter' to distinguish it from the model parameters. Then, using the determined relative weight, they estimated the slip distribution that maximized the posterior distribution. The ABIC-based source inversion method has been applied not only to the estimation of static slip models (e.g. Yabuki \& Matsu'ura 1992; Sagiya 1999; Fukahata et al. 2004; Nishimura

* Previously at: Disaster Prevention Research Institute, Kyoto University, Uji, Kyoto, Japan. 
et al. 2004), but also to the estimation of spatiotemporal slip models (e.g. Yoshida 1989; Ide et al. 1996; Sekiguchi et al. 2000; Asano et al. 2005; Yagi \& Fukahata 2011; Tanaka et al. 2014). In the ABIC-based source inversion, the hyperparameters are not random variables and are estimated from the data with ABIC. Therefore, this method is classified as an empirical Bayes method (e.g. George 1985; Carlin \& Louis 2000), also known as the maximum marginal likelihood method. In the empirical Bayes method, the prior distribution is 'empirically' provided by the data.

In contrast, the standard Bayesian method regards hyperparameters as random variables as well as model parameters (e.g. Carlin \& Louis 2000; Beck 2010). This method is often called the 'fully' Bayesian method to distinguish it from the empirical Bayes method and this naming system is also employed in this paper. In the fully Bayesian method, the prior distributions of the hyperparameters are set before any data are observed. Then, the posterior distributions of both the model parameters and hyperparameters are directly estimated using a sampling method. From their obtained posterior distributions, the optimal values and uncertainties of the model parameters and hyperparameters can be estimated. The fully Bayesian method has recently found applications in various fields including geophysics because of the recent improvement in sampling methods and the availability of powerful computers. This method has also been used in earthquake source process analysis (e.g. Fukuda \& Johnson 2008; Monelli \& Mai 2008; Monelli et al. 2009; Fukuda \& Johnson 2010; Minson et al. 2013; Dettmer et al. 2014; Duputel et al. 2014; Minson et al. 2014; Razafindrakoto \& Mai 2014; Wang et al. 2015).

As stated by Backus (1988), every geophysical inverse problem involves an existence half (finding at least one plausible model that fits the data) and a uniqueness half (finding all plausible models that fit the data). The optimization method, which uses regularization with some constraints and obtains one optimal solution, solves the existence half of the inverse problem but cannot address the uniqueness half. In the empirical Bayes method, this method produces model-parameter distributions that follow Gaussian distributions because of its assumptions. From the distributions obtained by the empirical Bayes method, it is not possible to know the true distribution shapes and whether the Gaussian distribution assumption is appropriate. For hyperparameters, the empirical Bayes method determines only one optimal value; hence, this method does not yield any information about the hyperparameter distributions. Therefore, the empirical Bayes method cannot produce much information regarding the uniqueness half. On the other hand, the fully Bayesian method can generate distributions of model parameters and hyperparameters because sampling methods such as the Markov chain Monte Carlo (MCMC) method are used to directly obtain the posterior probability distribution. These distributions are useful for evaluating the uniqueness and reliability of the derived model. In this regard, fully Bayesian source inversion is preferable to ABIC-based source inversion.

Another advantage is that fully Bayesian source inversion can easily determine the appropriate hyperparameters when a non-negative slip constraint is used. Non-negative constraints have been widely used in previous source inversions to obtain physically reasonable slip models. Fukuda \& Johnson (2008) noted that the ABIC-based source inversion with a non-negative constraint does not work well in the determination of hyperparameters and typically generates a significantly under-smoothed or over-smoothed slip model. This phenomenon was interpreted as follows. In ABIC calculations, the posterior probability function is integrated with respect to the model parameters to obtain the marginal likelihood for hyperparameters. If a non-negative constraint is used, the posterior probability function should be non-zero when all of the model parameters are non-negative and should be zero otherwise; however, this function cannot be integrated analytically (Fukuda \& Johnson 2008). Therefore, posterior probability functions that ignore a non-negative constraint have been used in most ABIC-based source inversions in which a non-negative constraint was used to solve the inversion problems. In this case, the calculated ABIC do not properly account for a non-negative constraint and hyperparameters determined by the calculated ABIC might not be appropriate. This would cause the situation noted by Fukuda \& Johnson (2008). These ABIC-based source inversions are called 'conventional' ABIC-based source inversions in this paper. On the other hand, the fully Bayesian method can easily account for a non-negative constraint because it does not require analytical integration of the posterior possibility function over the model parameters. Fukuda \& Johnson (2008) demonstrated the usefulness of fully Bayesian source inversions through static slip inversions.

For the reasons given above, it is meaningful to use the fully Bayesian method in source inversions, and several studies have introduced this method into source inversions to estimate both static slip models (e.g. Fukuda \& Johnson 2008; Wang et al. 2015) and spatiotemporal slip models (e.g. Monelli et al. 2009; Minson et al. 2014). However, these estimated spatiotemporal slip models were relatively simple because the studies in which the models were derived used the single-time-window method to parametrize the source time functions for the subfaults. The single-time-window method uses a predetermined functional shape as a source time function (e.g. Fukuyama \& Irikura 1986; Cohee \& Beroza 1994; Cotton \& Campillo 1995; Ji et al. 2002). The unknown parameters are the slip timings, amounts and durations, indicating that source inversions that employ the single-time-window method are nonlinear problems. Although the single-time-window method has advantages, such as a small number of unknowns, this has a significant disadvantage in that the shape of the source time function that can be expressed in the source inversion is limited. If the complex source processes of huge earthquakes are to be analysed, the single-time-window method might not be suitable because it cannot express complex source time functions.

Another method commonly used to parametrize source time functions is the multiple-time-window method (e.g. Olson \& Apsel 1982; Hartzell \& Heaton 1983; Ide et al. 1996; Sekiguchi et al. 2000). In this method, the source time function is discretized into a series of several time-windows (element functions) and the only unknown parameters are the slip amounts at subfaults on time windows, indicating that source inversions using the multiple-time-window method are linear problems. Therefore, the multiple-time-window method has high flexibility regarding the shape of the source time function, and would be preferable for source-process analyses of huge earthquakes with a complex source process. 
In this study, we developed a new source inversion method in which a fully Bayesian method is employed in the multiple-time-window source inversion. This is the first study to combine a fully Bayesian method with the multiple-time-window source inversion. We then applied the newly developed method to the 2011 Ibaraki-oki, Japan, earthquake $\left(M_{\mathrm{w}} 7.9\right)$ to demonstrate its usefulness. In addition, we compared the results of fully Bayesian source inversion with those of conventional ABIC-based source inversion and ascertained whether the problem with the determination of hyperparameters noted by Fukuda \& Johnson (2008) occurs in the estimation of spatiotemporal slip models based on waveform data. We also conducted synthetic tests to verify the newly developed source inversion method and to identify whether the hyperparameter-determination problem also occurs in ideal situations.

\section{FULLY BAYESIAN FORMULATION OF MULTIPLE-TIME-WINDOW SOURCE INVERSION}

We present the fully Bayesian formulation of the multiple-time-window source inversion. Here, the idea of Fukuda \& Johnson (2008), who developed the fully Bayesian linear static slip inversion with geodetic data, is extended to linear source inversion to estimated spatiotemporal slip models with waveform data.

\subsection{Bayes' theorem}

From Bayes' theorem (e.g. Carlin \& Louis 2000; Tarantola 2005; Beck 2010), the probability density function (PDF) of unknown parameters $\mathbf{x}$ given observed data $\mathbf{d}, p(\mathbf{x} \mid \mathbf{d})$ called the posterior PDF, is

$p(\mathbf{x} \mid \mathbf{d})=\frac{p(\mathbf{d} \mid \mathbf{x}) p(\mathbf{x})}{\int_{-\infty}^{\infty} p(\mathbf{d} \mid \mathbf{x}) p(\mathbf{x}) \mathrm{d} \mathbf{x}}$

where $p(\mathbf{x})$ is the PDF expressing the prior information about $\mathbf{x}$ and is called the prior PDF, $p(\mathbf{d} \mid \mathbf{x})$ is the PDF of $\mathbf{d}$ given $\mathbf{x}$ (the PDF of the observation equation) and describes the misfit between the observed data and forward predictions given $\mathbf{x}$ and $\int_{-\infty}^{\infty} p(\mathbf{d} \mid \mathbf{x}) p(\mathbf{x}) \mathrm{d} \mathbf{x}$ normalizes the posterior PDF. Because the denominator is independent of the unknown parameters $\mathbf{x}, p(\mathbf{x} \mid \mathbf{d})$ is proportional to the numerator:

$p(\mathbf{x} \mid \mathbf{d}) \propto p(\mathbf{d} \mid \mathbf{x}) p(\mathbf{x})$.

This equation indicates that the posterior PDF is derived from the product of the observation-equation PDF and the prior PDF.

\subsection{Probability distribution of the observation equation}

According to Aki \& Richards (1980), the dislocation at an earthquake fault is related to the displacement at the surface via the representation theorem (Maruyama 1963; Burridge \& Knopoff 1964),

$u_{n}(\mathbf{x}, t)=\int \mathrm{d} \tau \iint_{S} \Delta u_{p}(\boldsymbol{\xi}, \tau) c_{p q r s}(\boldsymbol{\xi}) G_{n r, s}(\mathbf{x}, t-\tau ; \boldsymbol{\xi}, 0) v_{q}(\boldsymbol{\xi}) \mathrm{d} S$,

where $u_{n}(\mathbf{x}, t)$ is the $n$th component of the displacement at point $\mathbf{x}$ and time $t, \Delta u_{p}(\xi, \tau)$ is the dislocation in the $p$ th direction at point $\xi$ and time $\tau, c_{p q r s}$ is the elastic constant tensor, $G_{n r, s}$ is the spatial derivative of Green's tensor representing the $n$th component of the displacement response at point $\mathbf{x}$ to a point dislocation in the $r$ th direction on a plane normal to the $s$ th direction at $\xi, S$ is the fault plane surface and $v_{q}$ is the $q$ th component of $\boldsymbol{v}$, the vector normal to $S$. To obtain the velocity $\dot{u}_{n}(\mathbf{x}, t)$ at point $\mathbf{x}$ and time $t$, eq. (3) can be rewritten as

$\dot{u}_{n}(\mathbf{x}, t)=\int \mathrm{d} \tau \iint_{S} \Delta u_{p}(\boldsymbol{\xi}, \tau) c_{p q r s}(\boldsymbol{\xi}) \dot{G}_{n r, S}(\mathbf{x}, t-\tau ; \xi, 0) v_{q}(\boldsymbol{\xi}) \mathrm{d} S$,

where $\dot{G}_{n r, s}$ is the temporal derivative of $G_{n r, s}$.

The fault plane can be parametrized into $n s$ smaller subfaults. The slip history of each subfault can also be parametrized into $n t m$ time windows following the multiple-time-window method. Eq. (4) can be discretized as

$\dot{u}_{n}(\mathbf{x}, t)=\sum_{i t m=1}^{n t m} \sum_{i s=1}^{n s} \sum_{i r=1}^{n r} m(i r, i s, i t m) G(i r, i s, i t m)$,

$G(i r, i s, i t m)=\int \Delta u_{\text {unit }_{i r}}\left(\tau-T_{\text {trig }}\right) c_{p(i r) q r s}(\xi(i s)) \dot{G}_{n r, s}(\mathbf{x}, t-\tau ; \boldsymbol{\xi}(i s), 0) v_{q}(\xi(i s)) \mathrm{d} \tau$,

$T_{\text {trig }}=\frac{R_{i s}}{V_{\mathrm{ftw}}}+\Delta t w \cdot(i t m-1)$

where $m(\mathrm{ir}, \mathrm{is}, \mathrm{itm})$ is the amount of slip in the irth direction at the itmth time-window on the isth subfault, $n r$ is the number of slip directions (here, $n r$ is 2 ), $R_{i s}$ is the distance of the isth subfault from the rupture starting point along the assumed fault model, $\Delta t w$ is the interval of time 
windows, $\Delta u_{\text {unit }}$ is is the unit slip function and $V_{\mathrm{ftw}}$ is the triggering velocity of the first time-window. It should be noted that $V_{\mathrm{ftw}}$ is different from the rupture propagation velocity. The discretized observation equation with errors can be written in vector form as follows:

$\mathbf{G m}=\mathbf{d}+\boldsymbol{\epsilon}$,

where $\mathbf{G}$ is an $N \times M$ coefficient matrix whose elements are discretized-theoretical Green's functions from each time-window for each subfault at each station, $N$ and $M$ are the number of data and model parameters, respectively, $\mathbf{m}$ is an $M$-dimensional model parameter vector, d is an $N$-dimensional velocity data vector and $\epsilon$ is an $N$-dimensional error vector.

The errors $\epsilon$ contain observation errors and modelling prediction errors. In this study, we ignored the modelling errors. One reason for this was that the true structure of the modelling errors is unclear in source inversions using seismic waves. The other reason was that substantial calculation would be required to introduce the modelling errors into the fully Bayesian source inversion. As will be explained later, we use the MCMC method, which updates the unknown parameters in small increments, to obtain the posterior probability distributions of the unknown parameters. The introduction of modelling errors requires the determinant of the covariance matrix to be calculated every time the unknown parameters are updated (e.g. Yagi \& Fukahata 2011; Duputel et al. 2014). If this approach were used together with the MCMC method, enormous calculation cost would be required to achieve convergence of the Markov chain. Therefore, we determined that it would be excessively difficult to introduce the modelling errors in this study. For the observation errors, we assumed a Gaussian distribution and that the errors are independent from each other. In this case, the errors $\boldsymbol{\epsilon}$ follow a Gaussian distribution of mean $\mathbf{0}$ and covariance matrix $\sigma^{2} \mathbf{I}_{N}$, $\boldsymbol{\epsilon} \sim \mathcal{N}\left(\mathbf{0}, \sigma^{2} \mathbf{I}_{N}\right)$, where $\mathbf{I}_{N}$ is an $N$-dimensional identity matrix and $\sigma^{2}$ is an unknown scale factor for the covariance matrix. Then, eq. (8) can be expressed as the PDF:

$p\left(\mathbf{d} \mid \mathbf{m}, \sigma^{2}\right)=\left(2 \pi \sigma^{2}\right)^{-N / 2} \exp \left[-\frac{1}{2 \sigma^{2}}\|\mathbf{d}-\mathbf{G m}\|^{2}\right]$,

where $\|\mathbf{a}\|^{2}=\mathbf{a}^{T} \mathbf{a}$. When $p\left(\mathbf{d} \mid \mathbf{m}, \sigma^{2}\right)$ is viewed as a function of $\mathbf{m}$ given $\mathbf{d}$ and $\sigma^{2}, p\left(\mathbf{d} \mid \mathbf{m}, \sigma^{2}\right)$ is called the likelihood function. The unknown parameter $\sigma^{2}$ is called a hyperparameter.

\subsection{Prior probability distribution}

In order to obtain a stable solution, previous source-inversion studies commonly employed a slip smoothing constraint (e.g. Hartzell \& Heaton 1983; Ide et al. 1996; Sekiguchi et al. 2000; Kubo \& Kakehi 2013). When a spatiotemporal source model is estimated, a smoothing constraint is needed in space and time. Although some previous studies used different smoothing constraints in space and time (e.g. Ide et al. 1996), this approach cannot take into account the difference in rupture initiation times between adjacent subfaults. One way of overcoming this problem is to use the spatiotemporal distance between the unknown parameters. Herein, we present a spatiotemporal smoothing constraint that uses the derived spatiotemporal distance between model parameters $\mathbf{m}$ following Sekiguchi et al. (2000):

$$
\left\{\begin{array}{c}
\sum_{i^{\prime}} \frac{1}{r_{i-i^{\prime}}} \\
r_{i-i^{\prime}} \leq r_{0}
\end{array}\right\} m_{i}=\begin{aligned}
& \sum_{i^{\prime}} \frac{m_{i^{\prime}}}{r_{i-i^{\prime}}} \\
& r_{i-i^{\prime}} \leq r_{0}
\end{aligned}
$$

where $r_{i-i^{\prime}}$ is the spatiotemporal distance between the $i$ th and $i^{\prime}$ th unknown parameters:

$r_{i-i^{\prime}}=\left\{\left|T_{i s}-T_{i s^{\prime}}\right|+\left|i t m-i t m^{\prime}\right| \cdot \Delta t w\right\} \cdot V_{\mathrm{ftw}}+r_{i s-i s^{\prime}}^{\text {space }}$,

where $T_{i s}$ is the time of the rupture front arrival at the $i s t h$ subfault, $r_{i s-i s^{\prime}}^{\text {space }}$ is the spatial distance between the $i s t$ and $i s^{\prime}$ th subfaults and $i=(i s$ $-1) \cdot n t m+i t m$. Although Sekiguchi et al. (2000) used the $S$-wave velocity $V_{\mathrm{s}}$ to convert temporal distance to spatial distance, we employed the triggering velocity of the first time-window $V_{\mathrm{ftw}}$. This is because the propagation distance of the time-window over the fault plane during time $\Delta T$ is not $\Delta T \cdot V_{\mathrm{s}}$ but rather $\Delta T \cdot V_{\mathrm{ftw}}$, and so the smoothing weight of an unknown parameter with a temporal distance of $\Delta T$ should be equivalent to that of an unknown parameter with a spatial distance of $\Delta T \cdot V_{\mathrm{ftw}}$. The smoothing distance is limited by $r_{0}$, which is equivalent to twice the average subfault length, as was determined by a trial-and-error process.

The discretized smoothing constraint in the vector form becomes

$\mathbf{S m}=\delta$,

where $\mathbf{S}$ is an $M \times M$ matrix that represents the finite difference approximation of the smoothing constraint, and $\boldsymbol{\delta}$ is an $M$-dimensional vector that determines the smoothness of the slip distribution. We assumed $\boldsymbol{\delta}$ to follow a Gaussian distribution with mean $\mathbf{0}$ and covariance matrix $\alpha^{2} \mathbf{I}_{M}, \boldsymbol{\delta} \sim \mathcal{N}\left(\mathbf{0}, \alpha^{2} \mathbf{I}_{M}\right)$, where $\mathbf{I}_{M}$ is an $M$-dimensional identity matrix and $\alpha^{2}$ is an unknown scale factor that characterizes the magnitude of $\delta$. In this case, the smoothing constraint of eq. (12) can be expressed as the PDF:

$p\left(\mathbf{m} \mid \alpha^{2}\right)=\left(2 \pi \alpha^{2}\right)^{-M / 2}\left|\mathbf{S}^{T} \mathbf{S}\right|^{1 / 2} \exp \left[-\frac{1}{2 \alpha^{2}}\|\mathbf{S m}\|^{2}\right]$. 
We also introduced a non-negative slip constraint into the prior PDF following Fukuda \& Johnson (2008):

$p\left(\mathbf{m} \mid \alpha^{2}\right)= \begin{cases}\frac{1}{Z_{\text {prior }}}\left(2 \pi \alpha^{2}\right)^{-M / 2}\left|\mathbf{S}^{T} \mathbf{S}\right|^{1 / 2} \exp \left[-\frac{1}{2 \alpha^{2}} \| \mathbf{S} \mathbf{m}||^{2}\right] & \text { for } \forall m_{i} \geq 0, \\ 0 & \text { otherwise },\end{cases}$

where $Z_{\text {prior }}$ is a constant that is introduced to normalize $p(\mathbf{m} \mid \alpha)$ (i.e. $\left.\int_{-\infty}^{\infty} p\left(\mathbf{m} \mid \alpha^{2}\right) \mathrm{d} \mathbf{m}=1\right)$. This PDF is proportional to eq. (13) if the non-negative constraints are satisfied and it is zero otherwise.

\subsection{Posterior probability distribution}

Let $\mathbf{x}^{T}=\left[\mathbf{m}^{T}, \sigma^{2}, \alpha^{2}\right]^{T}$ be a vector of unknown parameters. Substituting this $\mathbf{x}$ into the Bayes' theorem formula shown in eq. (2) gives

$p\left(\mathbf{m}, \sigma^{2}, \alpha^{2} \mid \mathbf{d}\right) \propto p\left(\mathbf{d} \mid \mathbf{m}, \sigma^{2}, \alpha^{2}\right) p\left(\mathbf{m}, \sigma^{2}, \alpha^{2}\right)$,

where $p\left(\mathbf{d} \mid \mathbf{m}, \sigma^{2}, \alpha^{2}\right)$ is the observation-equation PDF and $p\left(\mathbf{m}, \sigma^{2}, \alpha^{2}\right)$ is the joint prior PDF of $\mathbf{m}, \sigma^{2}$ and $\alpha^{2}$. Because the observationequation PDF and the prior PDF of $\mathbf{m}$ are independent of $\alpha^{2}$ and $\sigma^{2}$, respectively, eq. (15) can be rewritten as

$p\left(\mathbf{m}, \sigma^{2}, \alpha^{2} \mid \mathbf{d}\right) \propto p\left(\mathbf{d} \mid \mathbf{m}, \sigma^{2}\right) p\left(\mathbf{m} \mid \alpha^{2}\right) p\left(\sigma^{2}, \alpha^{2}\right)$,

where $p\left(\sigma^{2}, \alpha^{2}\right)$ is a prior PDF of $\sigma^{2}$ and $\alpha^{2}$. In this study, $p\left(\sigma^{2}, \alpha^{2}\right)$ was assumed to be a uniform PDF. Substituting eqs (9) and (14) into eq. (16) yields the equation:

$p\left(\mathbf{m}, \sigma^{2}, \alpha^{2} \mid \mathbf{d}\right)= \begin{cases}\frac{1}{Z}\left(\sigma^{2}\right)^{-N / 2}\left(\alpha^{2}\right)^{-M / 2} \exp \left[-\frac{1}{2 \sigma^{2}}\|\mathbf{d}-\mathbf{G m}\|^{2}-\frac{1}{2 \alpha^{2}}\|\mathbf{S m}\|^{2}\right] & \text { for } \forall m_{i} \geq 0, \sigma^{2}>0, \alpha^{2}>0, \\ 0 & \text { otherwise }\end{cases}$

where $Z$ is a normalizing constant independent of $\mathbf{m}, \sigma^{2}$ and $\alpha^{2}$. Because $\sigma^{2}$ and $\alpha^{2}$ must be positive, $\Sigma=\log _{10} \sigma^{2}$ and $A=\log _{10} \alpha^{2}$ were sampled instead of $\sigma^{2}$ and $\alpha^{2}$ :

$p(\mathbf{m}, \Sigma, A \mid \mathbf{d})= \begin{cases}\frac{1}{Z}\left(10^{\Sigma}\right)^{-N / 2}\left(10^{A}\right)^{-M / 2} \exp \left[-\frac{1}{2 \cdot 10^{\Sigma}}\|\mathbf{d}-\mathbf{G m}\|^{2}-\frac{1}{2 \cdot 10^{A}}\|\mathbf{S m}\|^{2}\right] & \text { for } \forall m_{i} \geq 0, \\ 0 & \text { otherwise. }\end{cases}$

This formulation for $\sigma^{2}$ and $\alpha^{2}$ has another advantage. Although the jumping width of $\Sigma(A)$ used to generate the candidate model in the Metropolis-Hastings algorithm, which will be explained later, is independent of the current value of $\Sigma(A)$, the jumping width of $\sigma^{2}=10^{\Sigma}$ $\left(\alpha^{2}=10^{A}\right)$ depends on the current value of $\Sigma(A)$. Therefore, the jumping width of $\sigma^{2}\left(\alpha^{2}\right)$ becomes small when $\sigma^{2}\left(\alpha^{2}\right)$ is small, and vice versa.

\subsection{Posterior probability distribution sampling and source model estimation}

Analytical acquisition of the posterior probability distribution is difficult because it includes integration over unknown parameters (the denominator of the right-hand side in eq. 1). Instead of analytical estimation, the MCMC method (e.g. Carlin \& Louis 2000) has often been used in previous studies (e.g. Fukuda \& Johnson 2008; Monelli et al. 2009). The distribution of discrete samples obtained by the MCMC method can be regarded as an approximation of the stationary distribution of the Markov chain, or the posterior probability distribution. In this study, we used the Metropolis-Hasting algorithm (e.g. Metropolis et al. 1953; Hastings 1970), which is one MCMC method, to obtain the posterior probability distributions for $\mathbf{m}, \Sigma$ and $A$. We let $\mathbf{x}^{T}=\left[\mathbf{m}^{T}, \Sigma, A\right]^{T}$ be an $(M+2)$-dimensional vector of unknown parameters. The algorithm used to sample the posterior PDF $p(\mathbf{x} \mid \mathbf{d})$ was as follows:

(1) The counter is set at $t=0$ and the initial state $\mathbf{x}^{(0)}$ is generated.

(2) A candidate $x_{i}^{\prime}$ is generated for $x_{i}^{(t)}$, which is one component of $\mathbf{x}^{(t)}$, from the proposal distribution $q\left(x_{i}^{\prime} \mid x_{i}^{(t)}\right) \sim \mathcal{U}\left(x_{i}^{(t)}-\Delta x_{i}, x_{i}^{(t)}+\Delta x_{i}\right)$ where $\mathcal{U}\left(x_{i}^{(t)}-\Delta x_{i}, x_{i}^{(t)}+\Delta x_{i}\right)$ is a 1-D uniform distribution over the interval $\left[x_{i}^{(t)}-\Delta x_{i}, x_{i}^{(t)}+\Delta x_{i}\right]$ and $\Delta x_{i}$ scales the jumping size of the Markov chain along the $i$ th coordinate direction in the parameter space. Then, a candidate state $\mathbf{x}^{\prime}$ is defined as a vector of unknown parameters in which $x_{i}^{(t)}$ is replaced by $x_{i}^{\prime}$ in $\mathbf{x}^{(t)}$.

(3) The acceptance probability $P_{\text {accept }}=\min \left(1, \frac{p\left(\mathbf{x}^{\prime} \mid \mathbf{d}\right)}{p\left(\mathbf{x}^{(t)} \mid \mathbf{d}\right)}\right)$ is computed using eq. (18).

(4) A random number $u$ is generated from a uniform distribution over the interval $[0,1], \mathcal{U}(0,1)$.

(5) If $P_{\text {accept }} \geq u$, the candidate state $\mathbf{x}^{\prime}: \mathbf{x}^{(t)}=\mathbf{x}^{\prime}$ is accepted. If $P_{\text {accept }}<u$, the candidate state is rejected and the current state is retained.

(6) For all of the unknown parameters, steps $2-6$ are repeated.

(7) The counter is increased from $t$ to $t+1: \mathbf{x}^{(t+1)}=\mathbf{x}^{(t)}$, and $\mathbf{x}^{(t+1)}$ is outputted for the $(t+1)$ th sample. Then, the algorithm returns to step 2 .

This algorithm is shown as a flow chart in Fig. 1.

For the initial state of the Markov chain, $\mathbf{x}^{(0)}$, we generated an random slip distribution $\mathbf{m}^{(0)}$ and random values of the hyperparameters $\left(\Sigma^{(0)}, A^{(0)}\right)$ from the uniform distributions $\mathcal{U}(0,1)$ and $\mathcal{U}(-1,0)$, respectively. The width of the proposal probability density $\Delta x_{i}$ controls the efficiency of the Metropolis algorithm and the acceptance rate in step 5. Gelman et al. (1996) suggested that the width of the proposal 


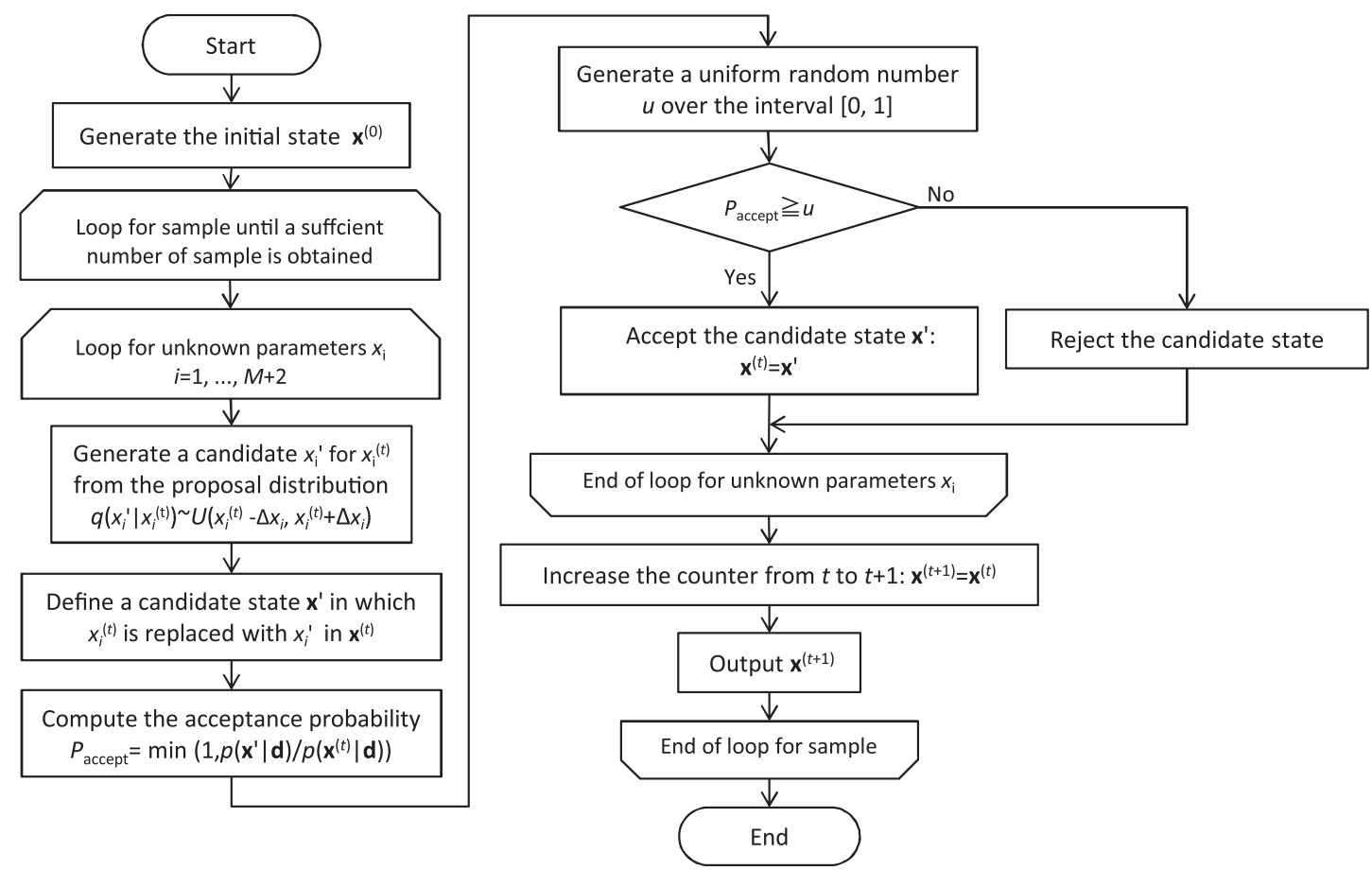

Figure 1. Flow chart of algorithm for sampling posterior possibility distribution function $p(\mathbf{x} \mid \mathbf{d})$.

probability density should be selected so that the acceptance rate of the Metropolis algorithm is approximately 44 per cent if there is only one model parameter and decreases to 23 per cent as the number of model parameters increases. In this study, we tested $\Delta x_{i}$ values by trial-and-error and chose the values that caused the acceptance rates of $\mathbf{m}, \Sigma$ and $A$ to be close to 23,44 and 44 per cent, respectively.

As the optimal values of the unknown parameters, we used the medians of their marginal posterior PDFs. Their uncertainties were evaluated by using the 2.5th and 97.5th percentiles of their marginal posterior PDFs. The marginal posterior PDFs for the unknown parameters were obtained as follows. Given a set of $N_{s}$ samples of $\mathbf{x},\left\{\mathbf{x}_{1}, \mathbf{x}_{2}, \ldots, \mathbf{x}_{N_{s}}\right\}$, which can be regarded as a set of samples drawn from the posterior PDF $p(\mathbf{x} \mid \mathbf{d})=p(\mathbf{m}, \Sigma, A \mid \mathbf{d})$, this set is equivalent to approximating the posterior PDF with the samples:

$p(\mathbf{x} \mid \mathbf{d}) \cong \frac{1}{N_{s}} \sum_{j=1}^{N_{s}} \delta\left(\mathbf{x}-\mathbf{x}_{j}\right)$,

where $\delta(\mathbf{x})$ is the Dirac delta function. Because a sample $\mathbf{x}_{j}$ can be denoted as $\mathbf{x}_{j}^{T}=\left[\mathbf{m}_{j}^{T}, \Sigma_{j}, A_{j}\right]$, the posterior PDF (19) can be expressed as

$p(\mathbf{m}, \Sigma, A \mid \mathbf{d}) \cong \frac{1}{N_{s}} \sum_{j=1}^{N_{s}} \delta\left(\mathbf{m}-\mathbf{m}_{j}\right) \delta\left(\Sigma-\Sigma_{j}\right) \delta\left(A-A_{j}\right)$.

The PDF of $\mathbf{m}$ given $\mathbf{d}, p(\mathbf{m} \mid \mathbf{d})$, which is called the marginal posterior PDF of $\mathbf{m}$, can be computed by marginalizing the posterior PDF $p(\mathbf{m}, \Sigma, A \mid \mathbf{d})$ over $\Sigma$ and $A$ :

$p(\mathbf{m} \mid \mathbf{d})=\int_{-\infty}^{\infty} \int_{-\infty}^{\infty} p(\mathbf{m}, \Sigma, A \mid \mathbf{d}) \mathrm{d} \Sigma \mathrm{d} A \cong \frac{1}{N_{s}} \sum_{j=1}^{N_{s}} \delta\left(\mathbf{m}-\mathbf{m}_{j}\right)$.

The marginal posterior PDFs of $\Sigma$ and $A$ can be obtained similarly.

The early stage of a Markov chain depends on the initial state $\mathbf{x}^{(0)}$ and should not be used to estimate the posterior probability distribution of $\mathbf{x}$. Therefore, we discarded the samples for this stage (called the burn-in stage) and used the subsequent samples to obtain the posterior probability distribution only after the Markov chains converged. In order to assess the convergence, we used trace plots of the residuals of the waveform fit and the smoothness. Moreover, we also used the convergence diagnosis method proposed by Geweke (1992), in which the equality of the means of the first 10 per cent and last 50 per cent of a Markov chain is investigated.

\section{APPLICATION TO THE 2011 IBARAKI-OKI EARTHQUAKE}

We applied the newly developed fully Bayesian multiple-time-window source inversion method to real data from the 2011 Ibaraki-oki earthquake. This earthquake occurred off the east coast of Ibaraki prefecture, Kanto, in south-eastern Japan at 15:15 on 2011 March 11, Japan 
Standard Time (06:15 on March 11, coordinated universal time). The moment magnitude $\left(M_{\mathrm{w}}\right)$ estimated from the centroid moment tensor (CMT) inversion by the Global CMT (GCMT) Project was 7.9. According to hypocentral information such as the location and the source mechanism, this event was a thrust-type interplate earthquake in the subduction zone, in which the Pacific plate subducts beneath the North American plate. It was the largest aftershock of the 2011 Tohoku-oki earthquake $\left(M_{\mathrm{w}} 9.1\right)$ and occurred approximately 30 min after the main shock.

Kubo et al. (2013) attempted to estimate the source process of this earthquake using the conventional ABIC-based multiple-time-window source inversion method from strong-motion and geodetic data; however, they could not determine the appropriate hyperparameters such as the relative weight of the different types of data-sets and the weight of the smoothing constraint. Therefore, they had no choice but to determine the hyperparameters by a trial-and-error process.

\subsection{Application details}

We used three components of strong-motion waveforms from the 17 stations:10 KiK-net (Aoi et al. 2011) stations observed on the ground surface, six K-NET (Aoi et al. 2011) stations observed on the ground surface and one F-net (Okada et al. 2004) station observed in the vault (Fig. 2). Except for the F-net station YMZ, whose original data are a velocity waveform, the observed acceleration waveforms were numerically integrated into velocity in the time domain. The velocity waveforms were bandpass filtered from 5 to $50 \mathrm{~s}$ and were resampled in $0.5 \mathrm{~s}$ increments. The time length of the waveforms is $150 \mathrm{~s}$ (starting $10 \mathrm{~s}$ before $P$-wave arrival, which was carefully identified by visual inspection).

Green's functions were calculated by the 3-D finite difference method (FDM) using the 3-D velocity structure model, the Japan Integrated Velocity Structure Model (JIVSM; Koketsu et al. 2012). Although the original model has the lowest $S$-wave velocity of $350 \mathrm{~m} \mathrm{~s}{ }^{-1}$ in the shallowest layer, the model we used filled this layer with the second shallowest layer, which has an $S$-wave velocity of $500 \mathrm{~m} \mathrm{~s}^{-1}$, to ensure the stability of the wavefield simulation. Because the northeastern limit of the Philippine Sea plate in the JIVSM is located further north than that estimated by Uchida et al. (2009), the layers of the Philippine Sea plate were ignored in the calculation of Green's functions. For the 3-D FDM calculations, we used the Ground Motion Simulator (Aoi \& Fujiwara 1999), which adopts discontinuous grids and has fourth-order accuracy in space and second-order accuracy in time. The discontinuous grids were of two sizes: $300 \mathrm{~m}$ (horizontal) $\times 200 \mathrm{~m}(\mathrm{vertical})$ in the shallow region and $900 \mathrm{~m}$ (horizontal) $\times 600 \mathrm{~m}$ (vertical) in the deep region. The connection depth of these grids was set to $11.0 \mathrm{~km}$. Considering the lowest $S$-wave velocity and grid spacing, this simulation is valid at periods longer than approximately $3 \mathrm{~s}$. The FDM model space we used was $304 \mathrm{~km}(\mathrm{NOE}) \times 274 \mathrm{~km}(\mathrm{~N} 90 \mathrm{E}) \times 188 \mathrm{~km}$ (depth) (Fig. 2). Inelastic attenuation was introduced as proposed in Graves (1996) and a reference period for the $Q$-value was set to $5 \mathrm{~s}$. The depth of the centre of each subfault in the assumed fault model was located at the top grid of the oceanic crust layer of the Pacific Plate.

Based on the shape of the plate boundary in the JIVSM, a curved fault model was assumed. The fault model was divided into 144 subfaults of about $10 \mathrm{~km} \times 10 \mathrm{~km}$ (Fig. 2). This subfault size is set to be smaller than the $S$-wave wavelength at the shortest analysis

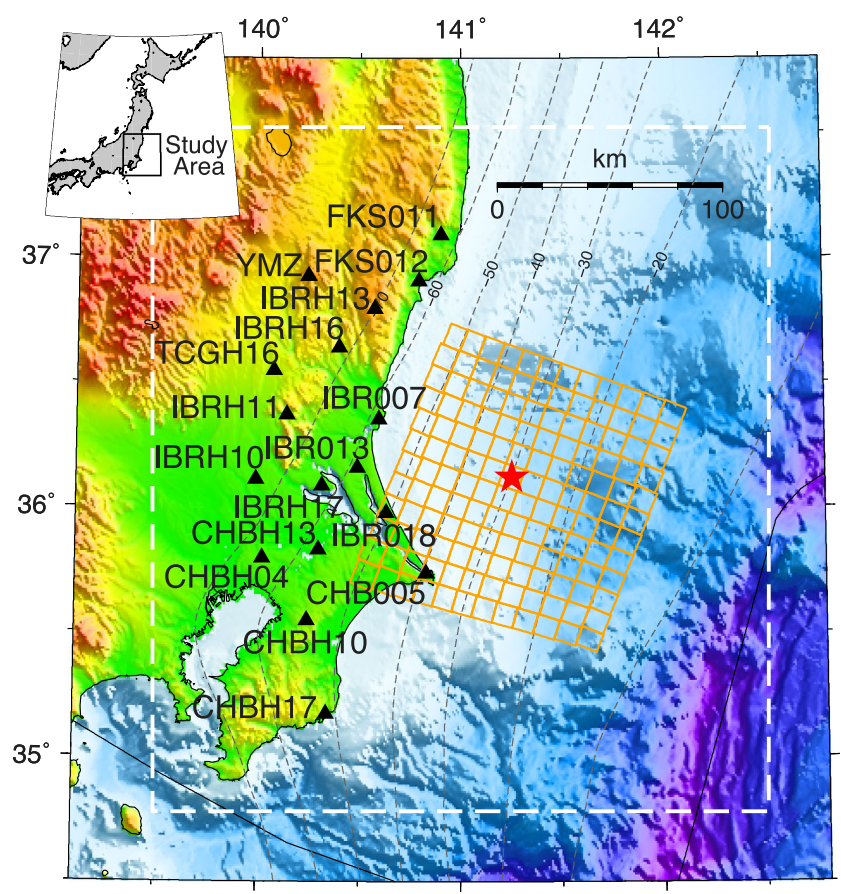

Figure 2. Map of stations (black triangles) used in this study. Red star is rupture starting point. Orange rectangular grid indicates assumed fault model. Broken white rectangle indicates horizontal extent of model space of finite difference method for calculation of 3-D Green's functions. 


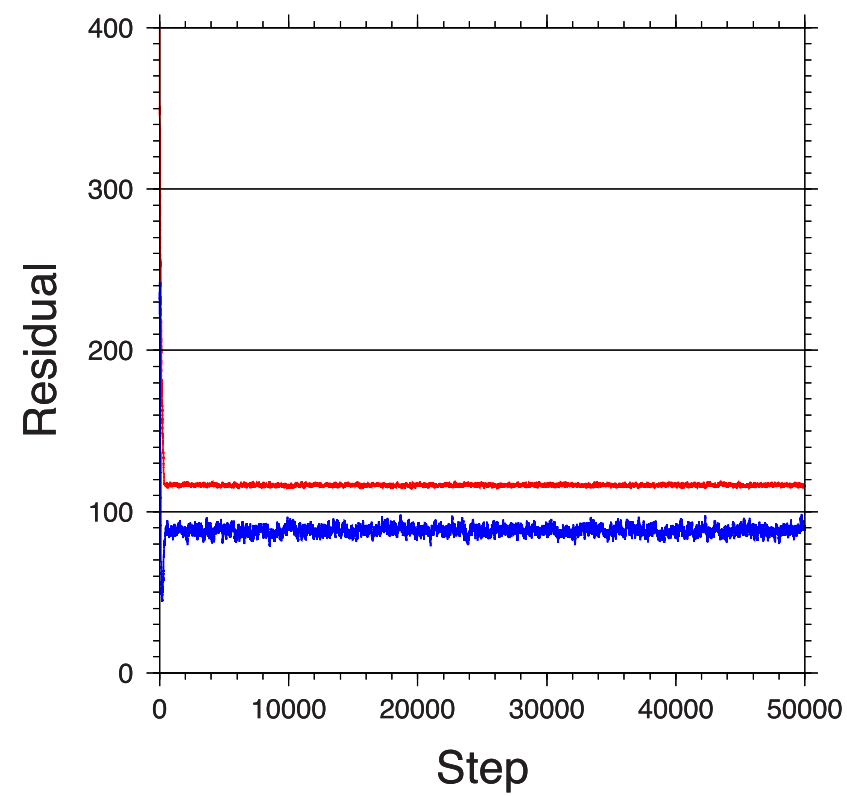

Figure 3. Trace plots of residuals of waveform fit (red) and smoothness (blue).

period ( 5 s). The strike and dip angles of each subfault were calculated by Shiono's (2007) method from grid data for the plate boundary of the JIVSM. The horizontal location of the rupture starting point was fixed at $\left(141.2525^{\circ} \mathrm{E}, 36.1208^{\circ} \mathrm{N}\right)$, which was the epicentre location in the JMA unified catalogue. The depth of the rupture starting point was set to $29.7 \mathrm{~km}$, corresponding to the depth of the plate boundary in the JIVSM at the JMA epicentre.

The slip time history at each subfault was represented by a series of nine smoothed-ramp functions with $4.0 \mathrm{~s}$ width, each with a $2.0 \mathrm{~s}$ lag. The duration of the smoothed-ramp functions was set to be shorter than the shortest analysis period ( $5 \mathrm{~s})$. The number of time-windows was determined by a trial-and-error process so that the source time function at each subfault would be properly explained. We estimated the weights of the slips with $45^{\circ}$ and $135^{\circ}$ rake angles for each time window at each subfault together with a non-negative constraint; hence the rake angle of the slip vector for each subfault was allowed to vary within $90^{\circ} \pm 45^{\circ}$. The triggering velocity of the first time-window of $2.0 \mathrm{~km} \mathrm{~s}^{-1}$ was selected to minimize the data fit residual.

The selected widths of the proposal probability densities for $\mathbf{m}, \Sigma$ and $A$ were $0.20,0.015$ and 0.040 , respectively. The sampling number in the Metropolis algorithm used in this application was 50000 . We discarded the first 10000 samples as burn-in samples. The subsequent 40000 samples were used to evaluate the optimal values of unknown parameters and their uncertainties. Fig. 3 shows the trace plots of the residuals of the waveform fit and the smoothness. The residuals are almost constant after the burn-in stage.

\subsection{Results}

Fig. 4 shows the marginal posterior probability distributions of the hyperparameters $(\Sigma, A)$ with the median and the 2.5 th and 97.5 th percentiles. The marginal posterior probability distributions are almost symmetric. The 95 per cent credible interval (CI, [97.5th percentile] [2.5th percentile]) for $A$ is larger than that for $\Sigma$, indicating that it is more difficult to constrain $A$ than it is to constrain $\Sigma$ in this application.

(a)

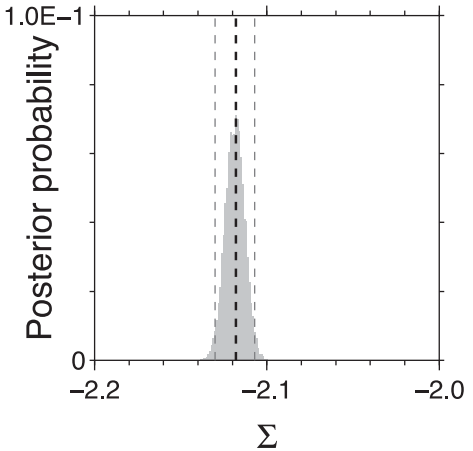

(b)

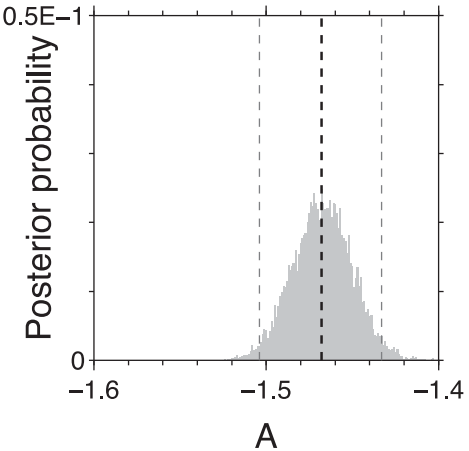

Figure 4. Marginal posterior probability distributions of (a) $\Sigma$ and (b) $A$ (solid histograms). Black and grey broken lines denote median and 2.5 th and 97.5 th percentiles of each sample distribution. 
Fig. 5 shows the marginal posterior probability densities of the slips $\mathbf{m}$ at each subfault in each time-window. Fig. 6 shows examples of the marginal posterior probability distributions of the slips $\mathbf{m}$ with the median and the 2.5 th and 97.5 th percentiles. At subfaults with some slips such as $(8,5)$, the marginal posterior probability distributions are uni-modal and nearly symmetric, which are similar to Gaussian distributions. On the other hand, at subfaults with slips close to zero such as $(4,10)$, the marginal posterior probability distributions are asymmetric and are truncated at zero because of the non-negative slip constraint. This indicates that the assumption of the empirical Bayes method that the posterior distributions for model parameters follow Gaussian distributions does not hold at subfaults with near-zero slips when a non-negative constraint is used.

(a)
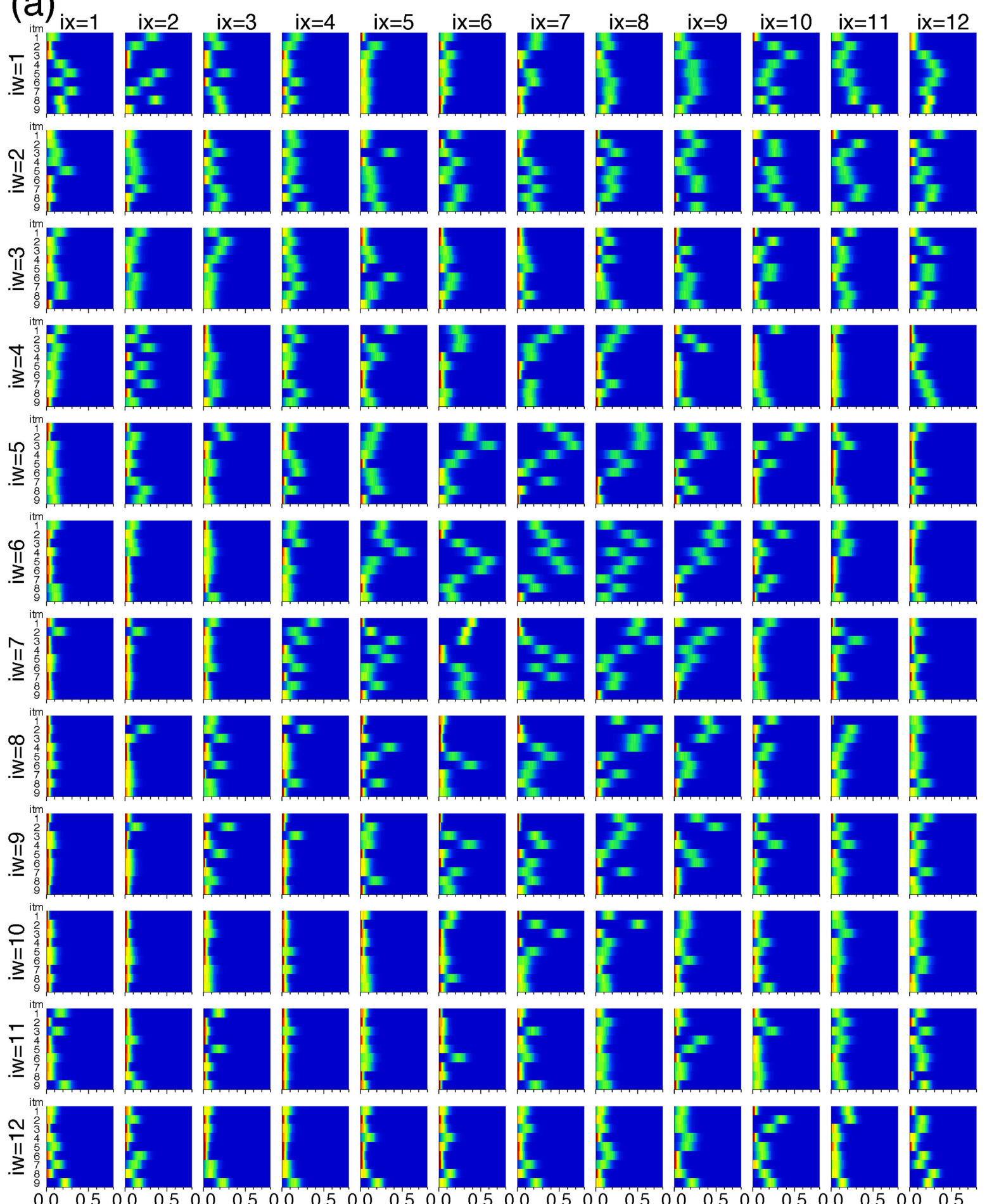

Figure 5. Marginal posterior probability densities of slips $\mathbf{m}$ at each subfault in each time-window with (a) $45^{\circ}$ and (b) $135^{\circ}$ rake angles. $i x$ and $i w$ are subfault numbers in strike and dip directions, respectively. itm is a time-window number. Subfault with the rupture starting point is located at $(i x, i w)=(6,7)$. 
Fig. 7 shows the distributions of the median, lower CI ([median] - [2.5th percentile]), coefficient of lower variance ([lower CI] / [median]), upper CI ([97.5th percentile] - [median]) and coefficient of upper variance ([upper CI] / [median]) for the slip with a $45^{\circ}$ of rake angle, the slip with a $135^{\circ}$ of rake angle, the magnitude of the synthetic slip vector, and its rake angle. Fig. 8 shows the moment rate function at each subfault for the median source model and the 2.5 th and 97.5 th percentile source models.

The estimated seismic moment and maximum slip of the optimal source model are $8.2 \times 10^{20} \mathrm{Nm}\left(M_{\mathrm{w}} 7.9\right)$ and $4.0 \mathrm{~m}$, respectively. A large slip area with the maximum slip is found from the hypocentre to $50 \mathrm{~km}$ south of the hypocentre (Fig. 7c). The other large slip area is located in the southeast fault edge (Fig. 7c). The lower and upper CIs are large in these areas (Figs 7e-g and 1-n); however, the coefficients of lower and upper variances in the southeast fault edge are larger than those in the peak slip area (Figs $7 \mathrm{i}-\mathrm{k}$ and $\mathrm{p}-\mathrm{r}$ ). It follows from this

(b)
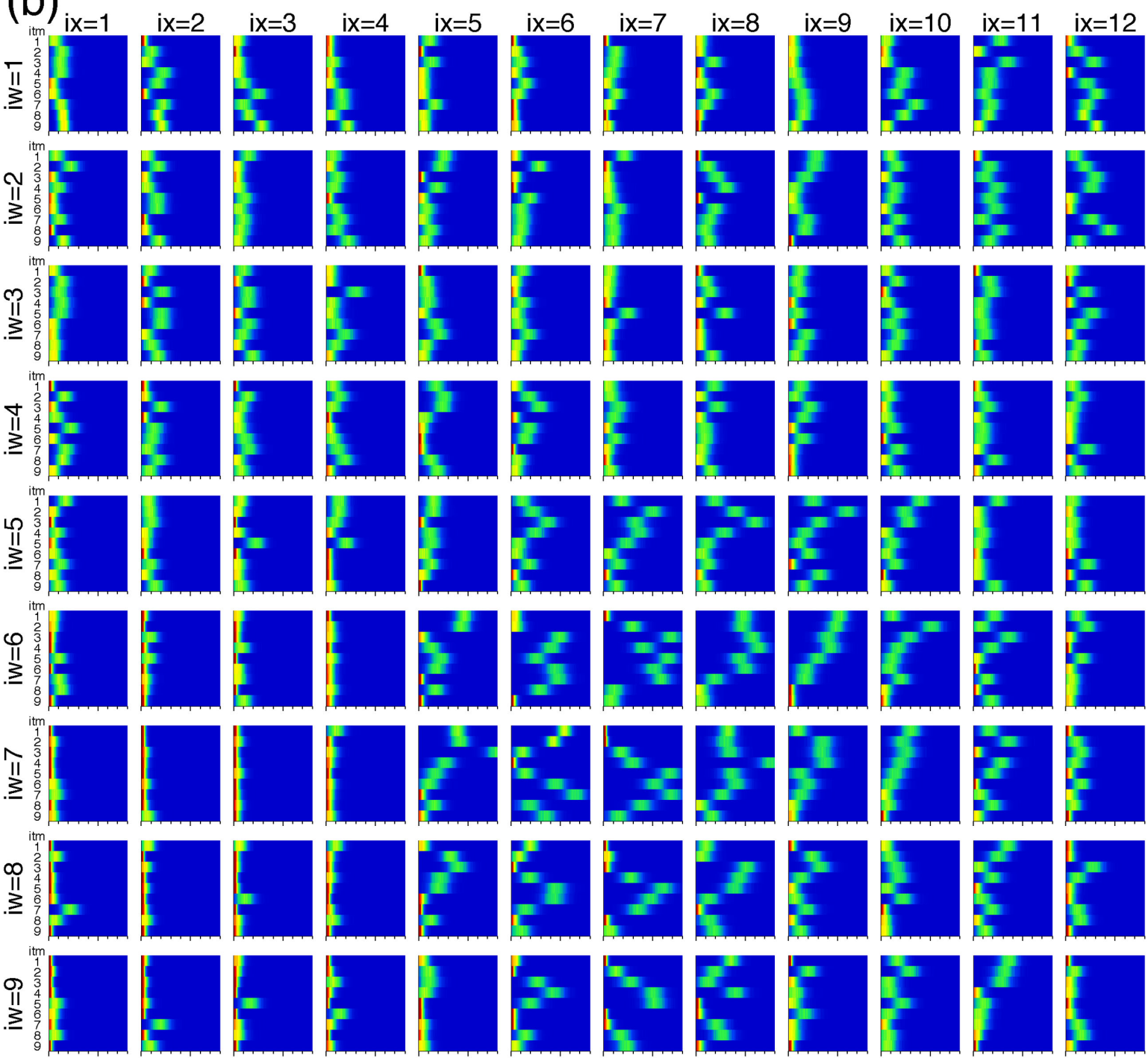

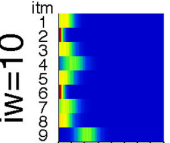
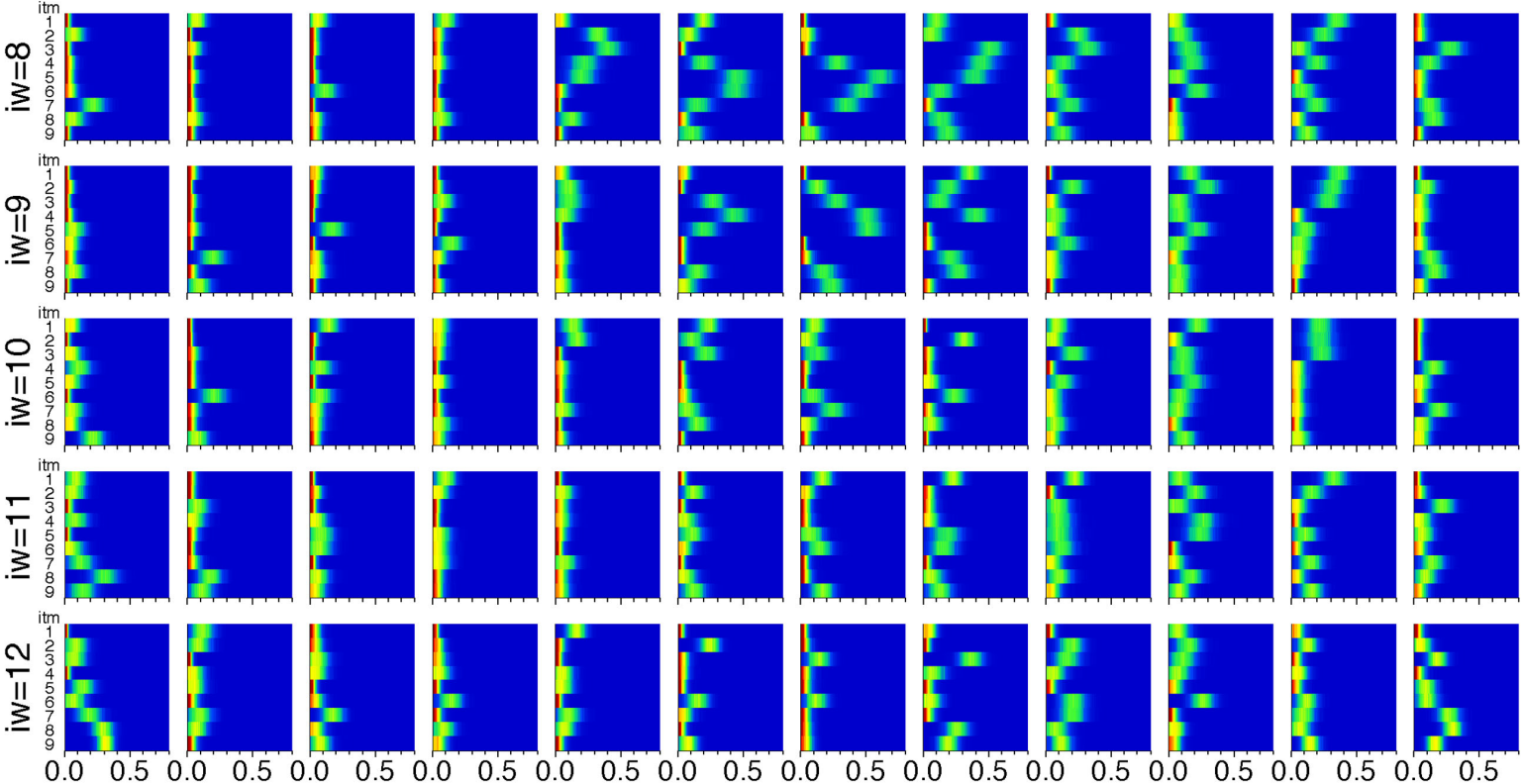

$\begin{array}{llll}0.0 & 0.5 & 0.0\end{array}$

Figure 5. (Continued.) 

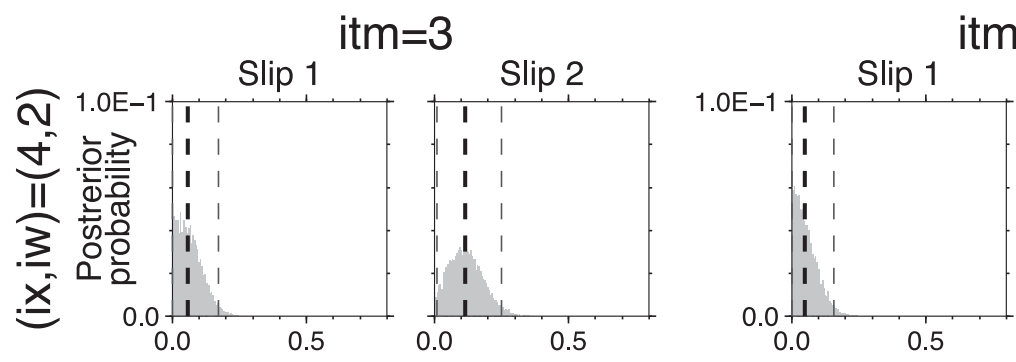

$\operatorname{tm}=6$
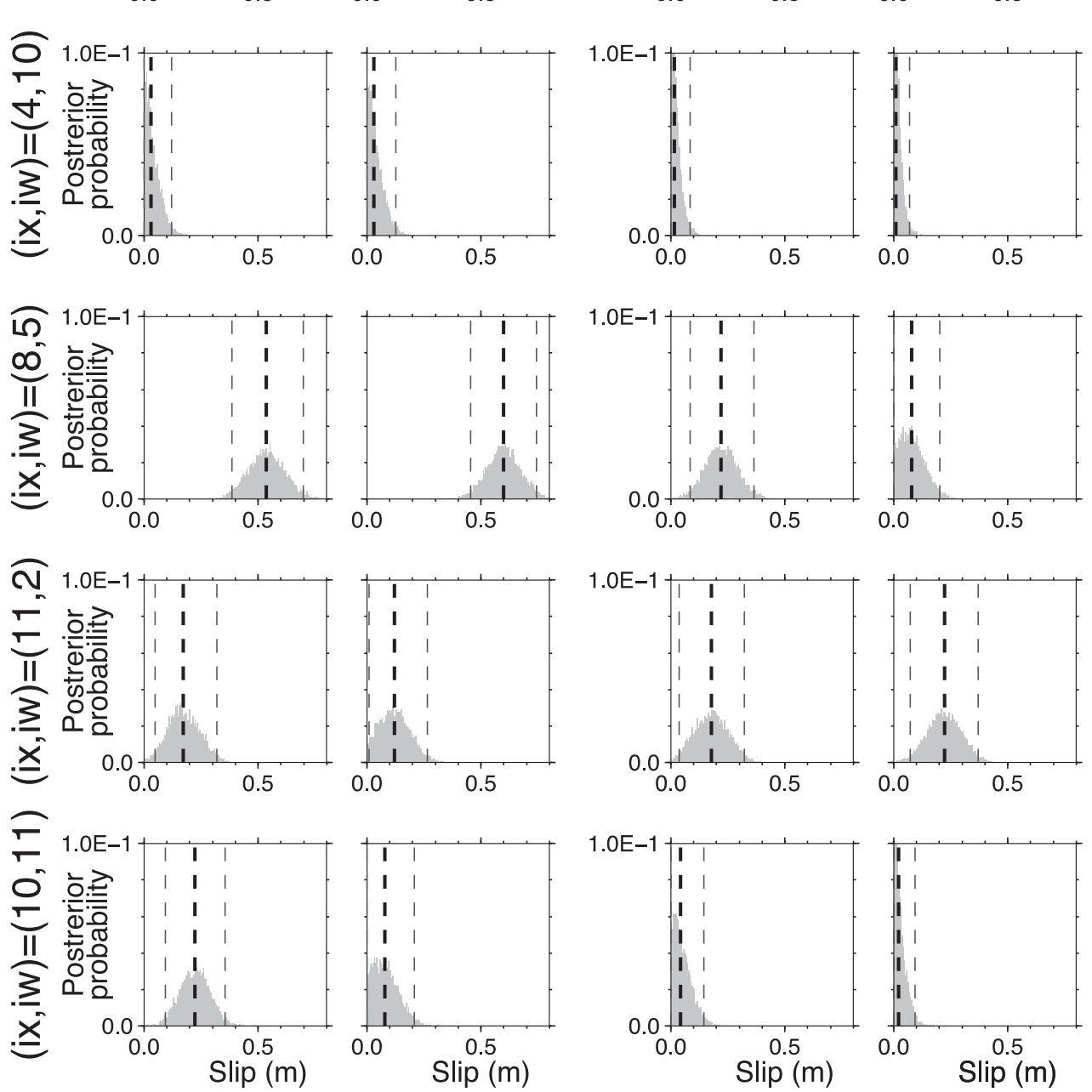

Figure 6. Examples of marginal posterior probability distributions of slips $\mathbf{m}$. Marginal posterior probability distributions of slips in subfaults $(i x, i w)=$ $(4,2),(4,10),(8,5)(10,2)$ and $(10,11)$ at time-window $\mathrm{itm}=3$ and 6 are shown. Black and grey broken lines denote median and 2.5 th and 97.5 th percentiles of each sample distributions.

that the slips in the southeast fault edge are expected to be relatively unreliable compared with those in the peak slip area. This might indicate that the rupture at the latter stage is relatively unconstrained, which is supported by the fact that the CI values are relatively large in the other fault edge (Figs $7 \mathrm{e}-\mathrm{g}$ and $1-\mathrm{n}$ ).

The lower CIs (Figs 7e-g) are smaller throughout the fault than the upper CIs (Figs 7l-n). This difference is considered to be caused by the use of a non-negative slip constraint. Its effect is significant in regions of small slip, such as subfaults $(4,10)$ and $(10,11)$.

In the northeast fault region where the slips are small, the variation of rake angle is large (Figs $7 \mathrm{~h}$ and o). In contrast, the variation of rake angle is small in the peak slip area (Figs $7 \mathrm{~h}$ and o). This difference indicates that the credibility of the estimated rake angle is related to the estimated slip amount.

The fit between the observed and synthetic strong-motion velocity waveforms is quite good (Fig. 9). The variance reduction is 82.4 per cent. Fig. 10 shows the synthetic waveforms at the KiK-net IBRH11 station that were produced by each sample of the slip model as well as the observed waveforms and the synthetic waveforms produced by the optimal slip model. This figure indicates that the variance of the waveform fit is not large among sample.

It is important to check whether the solution obtained by the fully Bayesian method is independent of its initial state. We conducted six runs of the Metropolis-Hasting algorithm using different random seeds to generate the initial states. Fig. 11(a) shows the first 5000 steps 
Slip 1
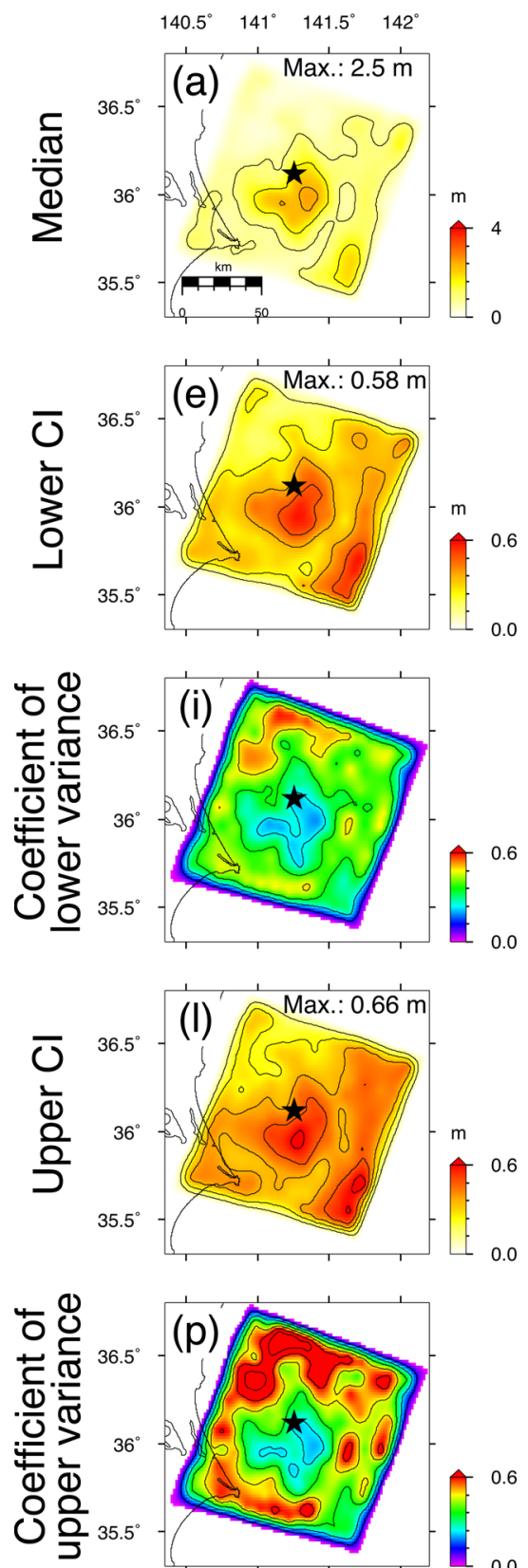

Slip 2

$140.5^{\circ} \quad 141^{\circ} \quad 141.5^{\circ} \quad 142^{\circ}$
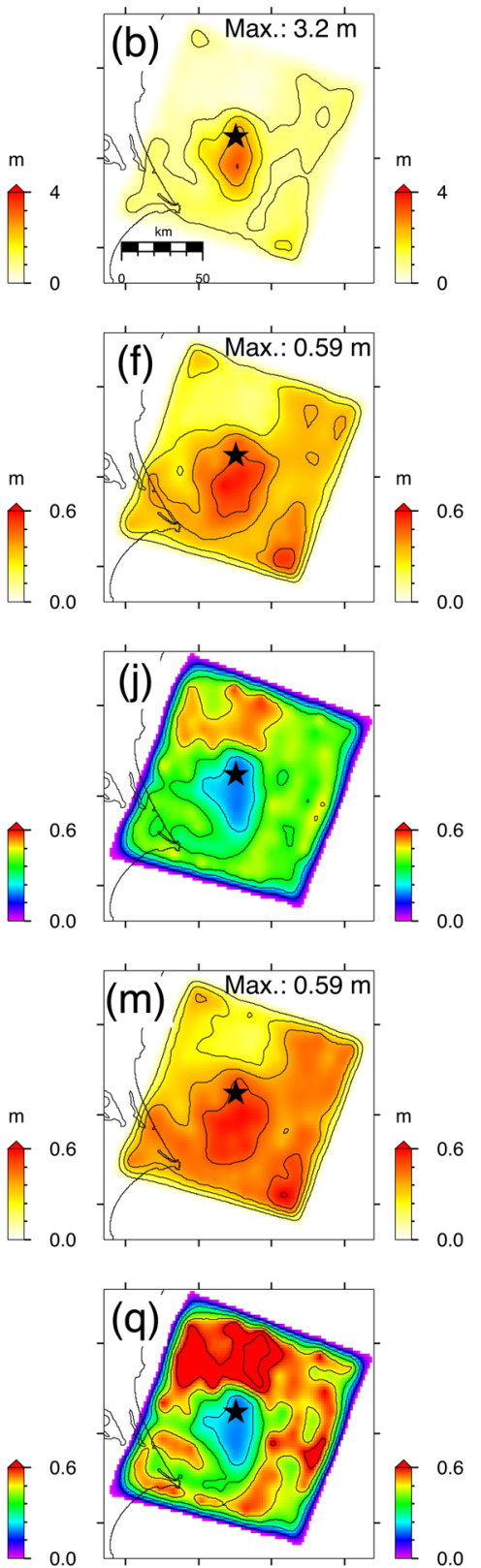

Synthetic slip
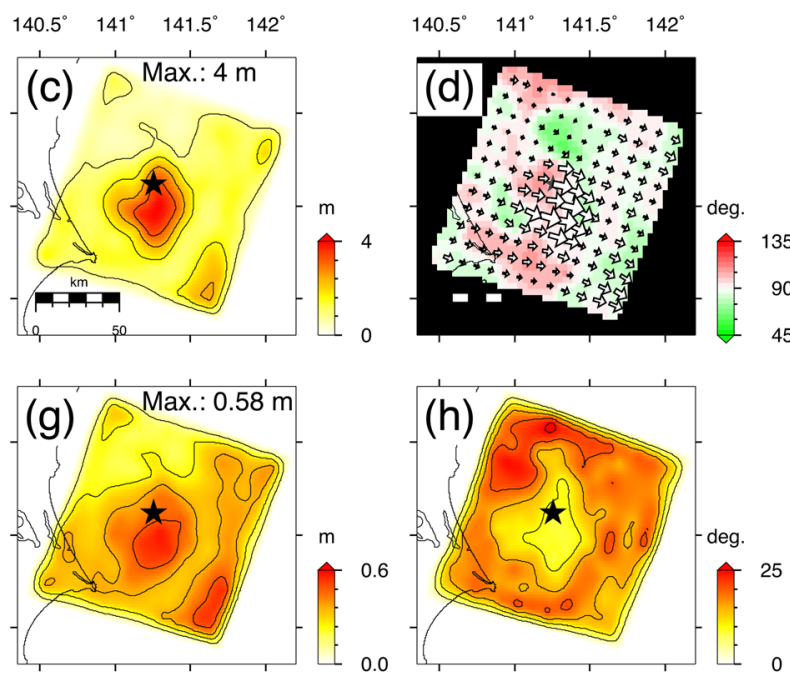

Figure 7. Distributions of medians (a, b, c, d), lower credible intervals ([median] - [2.5th percentile]) (e, $f, g, h)$, coefficients of lower variance ([lower credible interval] / [median]) $(\mathrm{i}, \mathrm{j}, \mathrm{k})$, upper credible intervals ([97.5th percentile] - [median]) $(1, \mathrm{~m}, \mathrm{n}, \mathrm{o})$ and coefficients of upper variance ([upper credible interval] / [median]) (p, q, r) for slip 1 with $45^{\circ}$ rake angle (a, e, i, l, p), slip 2 with $135^{\circ}$ rake angle (b, f, j, m, q), magnitude of synthetic slip vector (c, g, k, n, r), and its rake angle $(\mathrm{d}, \mathrm{h}, \mathrm{o})$. Solid stars indicate rupture starting point. Arrows in panel (d) indicate slip direction of hanging wall relative to foot wall. Common contours are used in $(a, b, c),(e, f, g, l, m, n),(h, o)$ and (i, j, k, p, q, r).

of trace plots for the residuals of waveform fit obtained by the six runs. Each residual of waveform fit converges to nearly the same value. Fig. 11(b) shows the final slip distributions of the source models obtained by the six runs. Fig. 11(c) shows the distributions of final-slip difference between the six source models in Fig. 11(b) and the source model in Fig. 7(c). These figures show that the estimated slip models are all nearly identical, and the difference in final slips among these source models is at the most $0.05 \mathrm{~m}$. These results means that the source model estimated by the newly developed source inversion method is independent of its initial state. The small difference among these source models indicates that the convergence of the source inversion with real data is very good in this study. This is expected to be because we used the Green's functions calculated with the latest 3-D velocity structure model and the realistic curved fault model based on the shape of the plate boundary. 


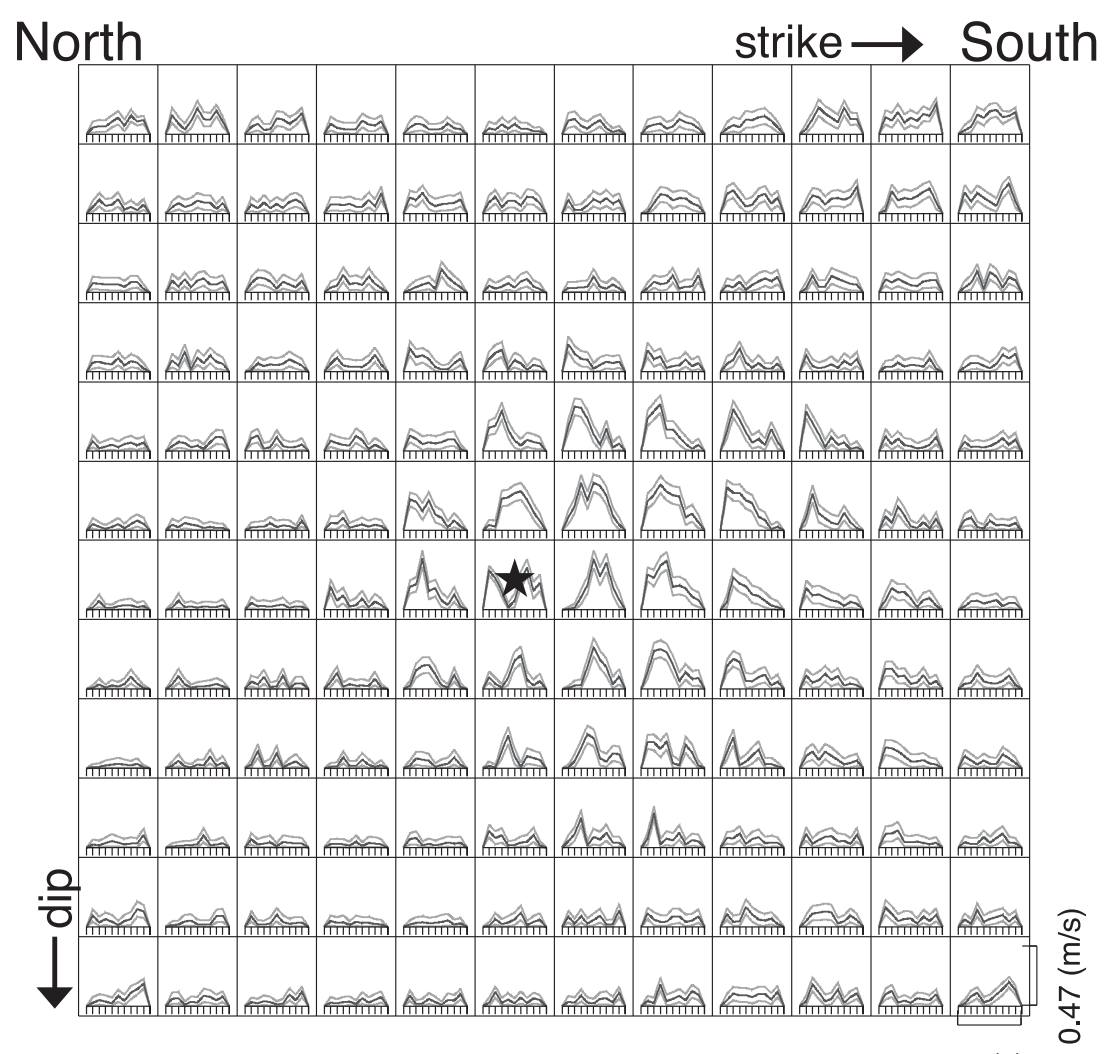

20 (s)

Figure 8. Estimated moment rate function at each subfault. Solid and broken lines indicate moment rate functions of median and 2.5th and 97.5th percentile source models. Solid star indicates subfault corresponding to rupture starting point.

\section{DISCUSSIONS}

For comparison of the results of the fully Bayesian source inversion, we also performed the conventional ABIC-based source inversion, which is similar to the method used by Sekiguchi et al. (2000). The inversion settings such as the smoothing constraint, fault model, station distribution and Green's functions were the same as those used for the fully Bayesian source inversion in this study. Fig. 12 shows the sample distribution of $p(\Sigma, A)$ obtained by the fully Bayesian method together with the optimal values of the hyperparameters estimated by the conventional ABIC-based method. The optimal value of $A$ obtained from the conventional ABIC-based method is larger than that obtained from the fully Bayesian method, although the values of $\Sigma$ are similar in both methods. This indicates that the relative weight of the waveform fit against the smoothing constraint is larger in the conventional ABIC-based method than it is in the fully Bayesian method. Fig. 13 shows the final slip distributions estimated by the fully Bayesian and conventional ABIC-based methods. The overall features of the final slip distribution estimated by the conventional ABIC-based method are comparable to those estimated by the fully Bayesian method (Fig. 13). However the maximum final slip estimated by the conventional ABIC-based method ( $8.3 \mathrm{~m})$ is far larger than that estimated by the fully Bayesian method $(4.0 \mathrm{~m})$, which reflects the differences in $(\Sigma, A)$. In addition, the seismic moment estimated by the conventional ABIC-based method $\left(1.5 \times 10^{21} \mathrm{Nm}\right)$ is much larger than that estimated by the fully Bayesian inversion, the JMA and the GCMT $\left(8.3 \times 10^{20}, 4.9 \times\right.$ $10^{20}$ and $8.5 \times 10^{20} \mathrm{Nm}$, respectively). The variance reduction in the conventional ABIC-based method (83.6 per cent) is larger than that in the fully Bayesian inversion ( 82.4 per cent). These results imply that the source model estimated by the conventional ABIC-based method is over-fitted and implausible, which corresponds with the conclusion of Fukuda \& Johnson (2008) that the conventional ABIC-based source inversion has a problem with the determination of hyperparameters when a non-negative constraint is used. Although Fukuda \& Johnson (2008) demonstrated this problem in static slip inversions, the result of this study indicates that this problem can also occur in waveform inversions.

In order to examine the verification of the fully Bayesian multiple-time-window source inversion and whether the results obtained using the fully Bayesian method differ from those of the conventional ABIC-based method even in the ideal situation, we conducted the following synthetic test. First, we prepared a simple source model consisting of a large-slip patch and a small-slip background region (Fig. 14a) which imitates the source model estimated with real data (Fig. 7c). The slip amount in each region and the area of the large-slip patch were assumed based on the empirical fault-parameter relationships for interplate earthquakes (Murotani et al. 2008). Next, we calculated two types of synthetic waveforms from the input slip model at the stations (Supporting Information Fig. S1): one had no noise and the other had Gaussian random noise with a 20 per cent standard deviation for the maximum amplitude of three components. Then, using these waveforms as the observed data, we estimated the source models using the fully Bayesian and conventional ABIC-based multiple-time-window source 


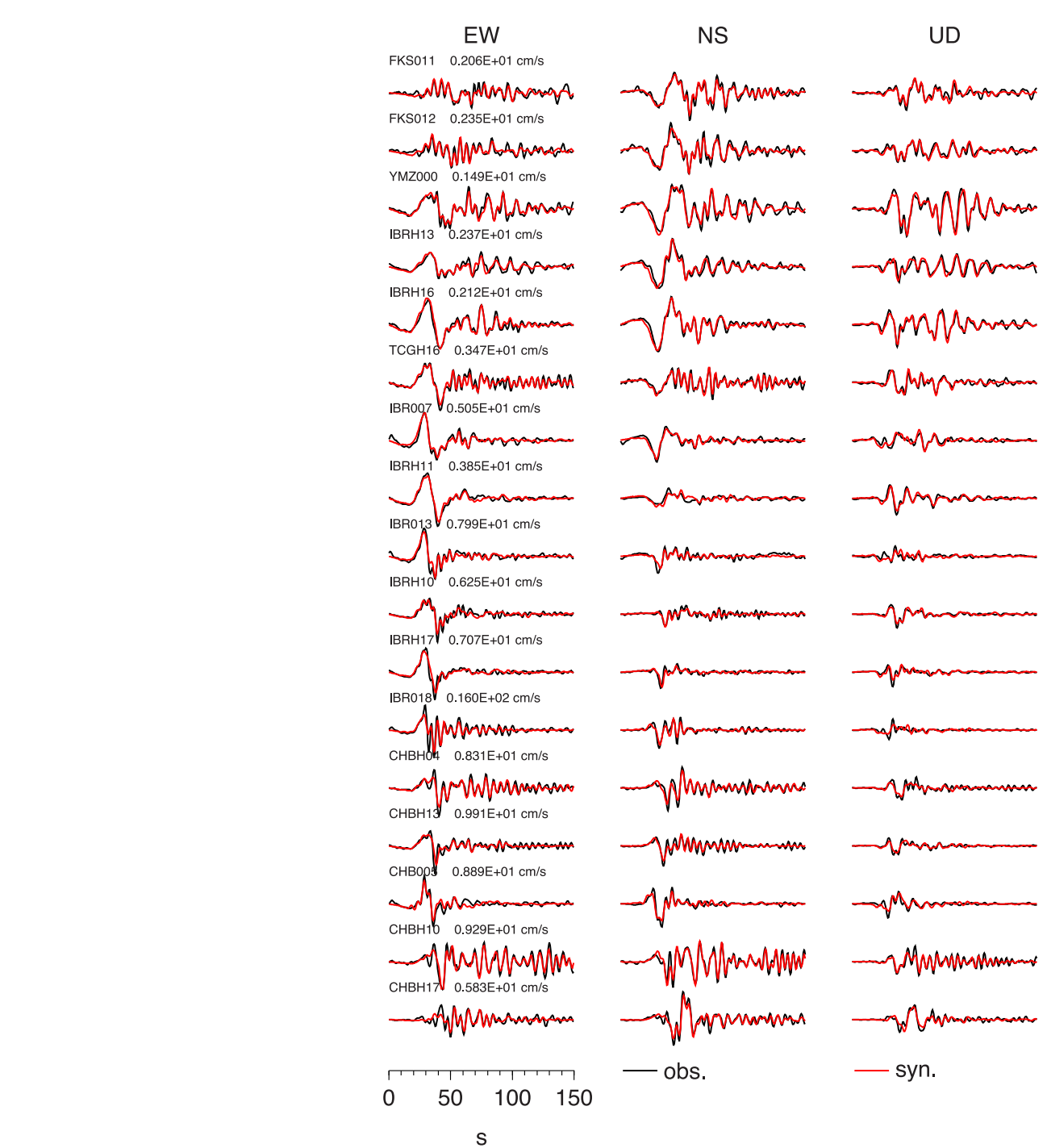

Figure 9. Comparison of observed (black) and synthetic (red) strong-motion velocity waveforms. Station name and maximum value of the waveforms are shown above each trace.

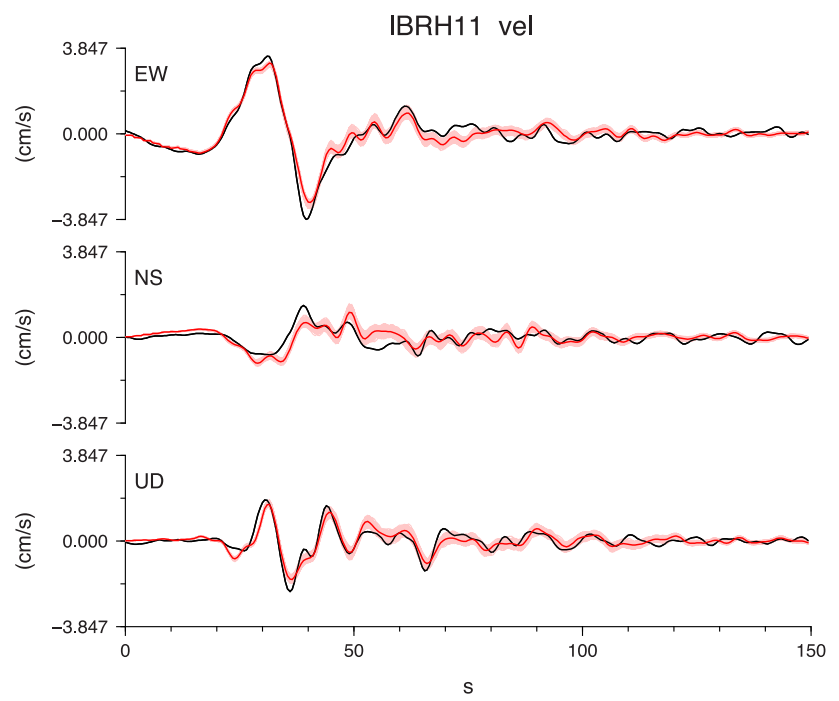

Figure 10. Comparisons of observed waveforms at KiK-net IBRH11 station (black), synthetic waveforms produced by each sample of source model (pink) and synthetic waveforms produced by optimal source model (red). 
(a)

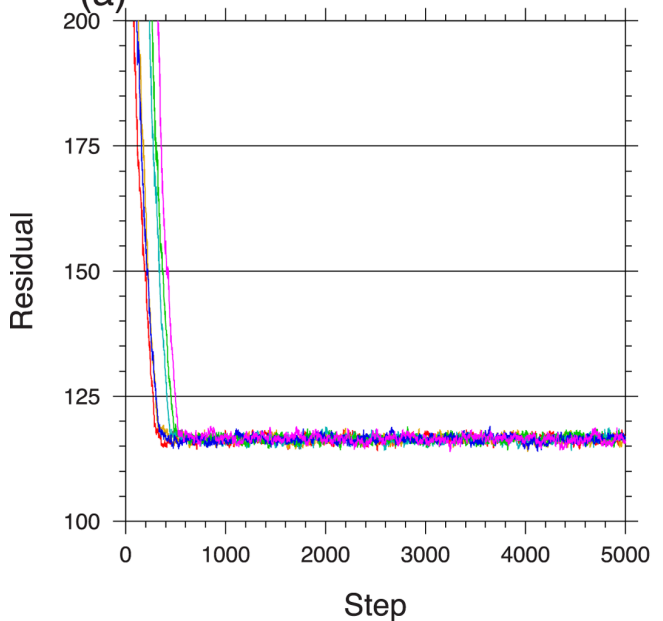

(b)
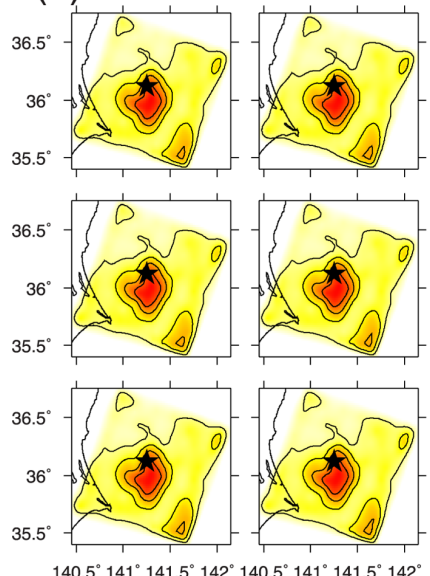

$140.5^{\circ} 141^{\circ} 141.5^{\circ} 142^{\circ} 140.5^{\circ} 141^{\circ} 141.5^{\circ} 142^{\circ}$

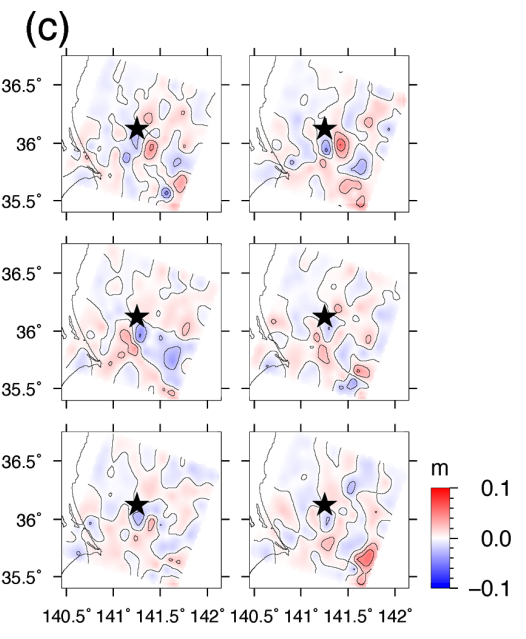

Figure 11. (a) First 5000 steps of trace plots for residuals of waveform fit obtained by six runs of the Metropolis-Hasting algorithm using different random seeds to generate initial states. Each colour represents one run of the Metropolis algorithm. (b) Final slip distributions obtained by the six runs of the Metropolis-Hasting algorithm. (c) Distributions of final-slip difference between the six source models in panel (b) and the source model in Fig. 7(c). Solid stars indicate rupture starting point.

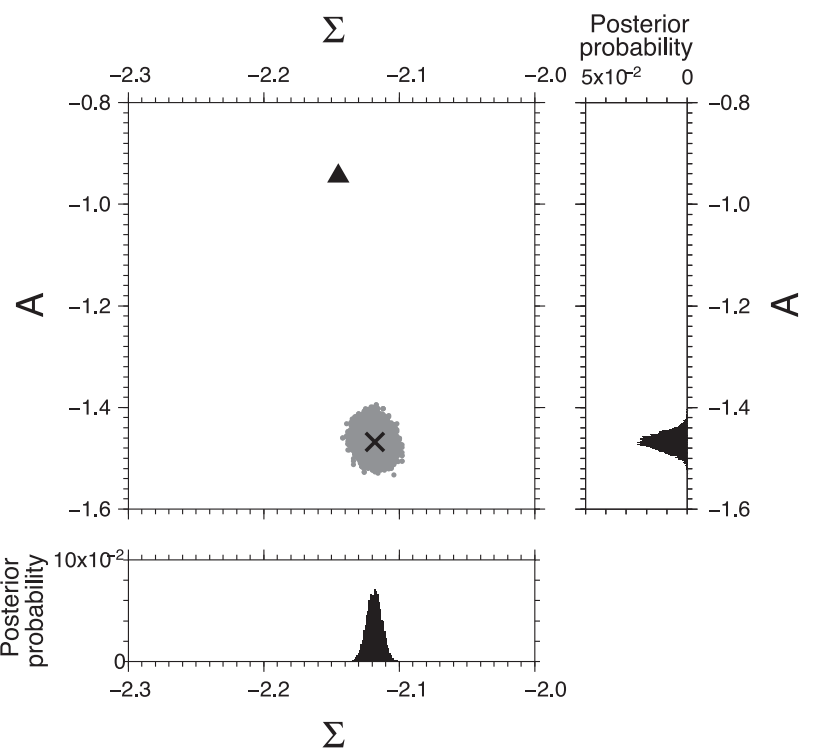

Figure 12. Sample distributions of $(\Sigma, A)$ obtained by fully Bayesian source inversion and marginal posterior probability distributions of $\Sigma$ and $A$. Samples are shown by grey dots. Cross indicates median of sample distributions of $\Sigma$ and $A$. Triangle indicates values of $\Sigma$ and $A$ estimated by conventional ABIC-based source inversion.

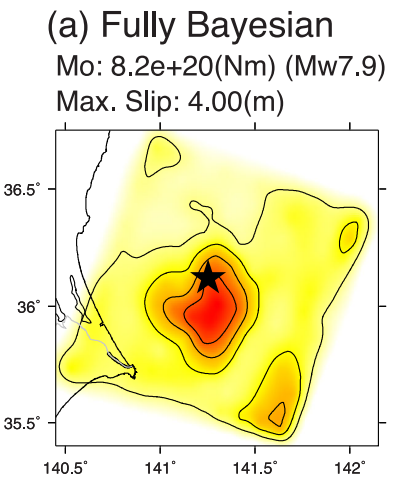

(b) Conventional ABIC

Mo: 1.5e+21(Nm) (Mw8.1)

Max. Slip: $8.30(\mathrm{~m})$

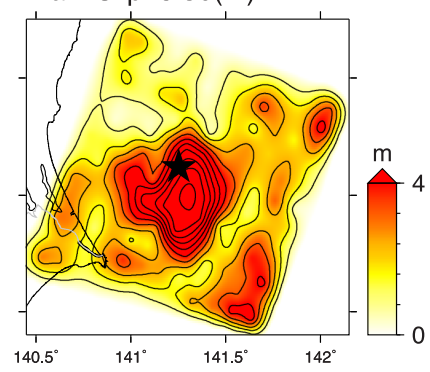

Figure 13. Final slip distributions of source models estimated by (a) fully Bayesian source inversion and (b) conventional ABIC-based source inversion. Solid stars indicate rupture starting point. 


\section{(a) Input model}

Mo: $8.6 e+20(\mathrm{Nm})$

Max. Slip: $3.00(\mathrm{~m})$

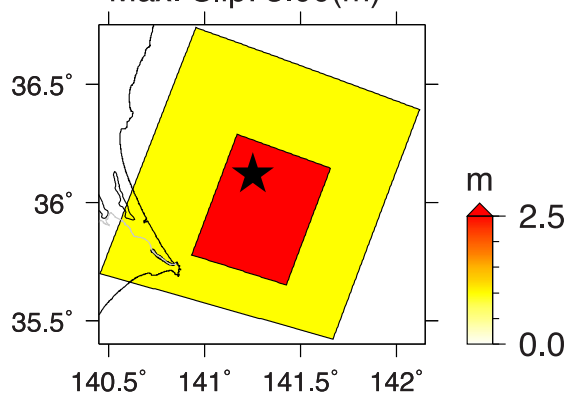

(b) Noise free

Fully Bayesian

Mo: $5.0 \mathrm{e}+20(\mathrm{Nm})$

Max. Slip: $2.20(\mathrm{~m})$

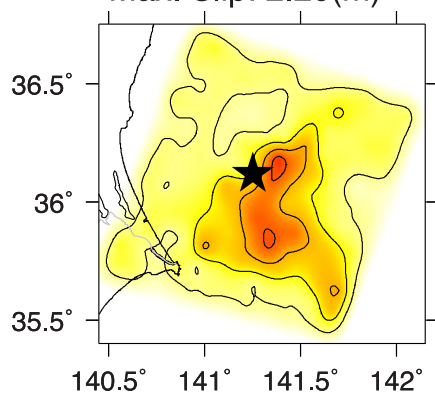

\section{$\mathrm{ABIC}$}

Mo: $1.0 \mathrm{e}+21(\mathrm{Nm})$

Max. Slip: $5.40(\mathrm{~m})$

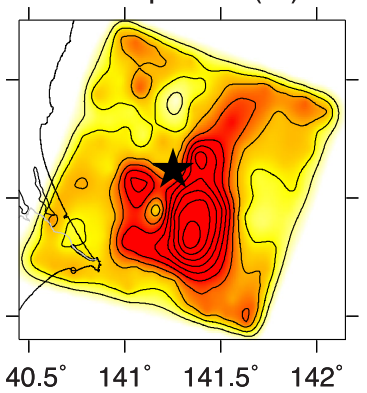

\section{(c) $20 \%$ Gaussian noise}

Fully Bayesian

Mo: $4.7 \mathrm{e}+20(\mathrm{Nm})$

Max. Slip: $1.80(\mathrm{~m})$

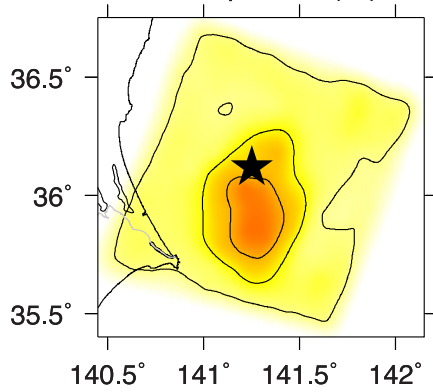

$\mathrm{ABIC}$

Mo: $9.6 \mathrm{e}+20(\mathrm{Nm})$

Max. Slip: $4.20(\mathrm{~m})$

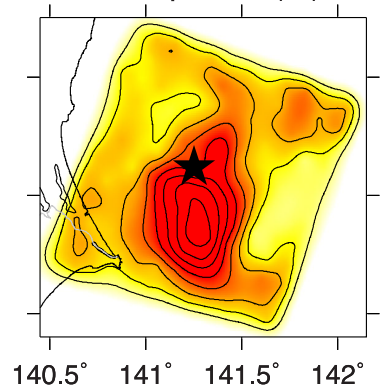

Figure 14. (a) Input slip model used for synthetic test, and results of synthetic tests in (b) noise-free case and (c) 20 per cent Gaussian noise case by (left) fully Bayesian source inversion and (right) conventional ABIC-based source inversion. Solid stars indicate rupture starting point.

inversions with the non-negative slip constraint. The inversion settings, such as the fault model and the station distribution, were the same as those used in the analysis of actual data. Figs 14(b) and (c) show the results of the synthetic tests. The fully Bayesian source inversion can resolve the large slip patch reasonably well not only in the noise-free case but also in the case of 20 per cent Gaussian noise. Although the maximum slip and seismic moment are underestimated in both cases, it is understandable because the one-sided station distribution such as that used in this study degrades the resolution of the source-inversion analysis. These results show that the newly developed source inversion method succeeds in producing a plausible source model. The conventional ABIC-based method produces solutions similar to those estimated by the fully Bayesian method. However, in the noise-free case, the maximum slip and seismic moment estimated by the conventional ABIC-based method are larger than those of the input model. In the case of 20 per cent Gaussian noise, the maximum slip and seismic moment are also overestimated. These results are expected to be caused by the use of the conventional ABIC-based source inversion together with a non-negative slip constraint. Although the overestimation in the case of 20 per cent Gaussian noise is not so large, this is considered to be caused by a coincidental balance between the abovementioned overestimation effect and the underestimation effect of the one-sided station distribution. The synthetic-test results presented herein are consistent of the actual results obtained using real data. 
We also conducted synthetic tests to investigate the spatial resolution of the fully Bayesian source inversion in the case of the 2011 Ibaraki-oki earthquake. In the synthetic tests of Supporting Information Fig. S2, the slip patterns of input models, which have two patches with the same slip amount, were recovered reasonably well, although the maximum slip and seismic moment were underestimated. In the synthetic tests of Supporting Information Fig. S3, input models were assumed to have two patches with different slip amounts, and the results show that the slip difference between the two patches can be resolved. These tests also indicate that the off-shore resolution is lower than the near-coast resolution (e.g. cases 3 and 4 in Supporting Information Fig. S2). Furthermore, we confirmed through the synthetic tests that the minimum residual of data fit is found when the triggering velocity of the first time window equals to that of the input one.

Thus, the synthetic tests in Fig. 14, and Supporting Information Figs S2 and S3 demonstrate that the fully Bayesian source inversion can resolve the overall feature of the source process for the 2011 Ibaraki-oki earthquake reasonably well. In their results, there are cases in which relatively large slips were found close to the southeast fault edge where large input slips were not assumed (e.g. Figs 14b and c). This implies that the slips in the southeast fault edge for the real source-inversion (Fig. 7) might be an artefact.

\section{CONCLUSIONS}

In this study, we developed a new source inversion method that combines a fully Bayesian method with the multiple-time-window source inversion. The introduction of a fully Bayesian method in source inversion has the following advantages: (1) When a non-negative constraint is used in the source inversion, the fully Bayesian source inversion can determine appropriate hyperparameters, whereas the conventional ABIC-based source inversion might not work well in the determination of hyperparameters. (2) Fully Bayesian source inversion produces the distributions of model parameters and hyperparameters that are useful in evaluating the uniqueness and reliability of a derived model. The use of the multiple-time-window method allows for flexibility in modelling the source time function at each subfault.

The developed source inversion method was applied to the 2011 Ibaraki-oki earthquake and the results showed the usefulness of this method. It was also demonstrated that the problem of the conventional ABIC-based method noted by Fukuda \& Johnson (2008) also occurs in the spatiotemporal slip inversion for this event and that the fully Bayesian source inversion can be used to overcome this problem. This trend was also observed in the synthetic tests.

Although this study showed that the conventional ABIC-based source inversion did not work well in the source-process analysis of the 2011 Ibaraki-oki earthquake, it does not mean that the problem of the conventional ABIC-based method 'generally' occurs. This study had the one-sided station distribution, and this would make it difficult and unstable to solve the source inversion. For discussing usefulness of the conventional ABIC-based method, we need further studies for other earthquakes, particularly earthquakes with a good station coverage.

\section{ACKNOWLEDGEMENTS}

We thank the anonymous reviewer and Prof Martin Mai for their helpful comments. We have benefited from discussions with Prof Yukitoshi Fukahata, Prof Junichi Fukuda, Prof Takaki Iwata and Prof Yuji Yagi. We used the strong-motion data recorded by K-NET, KiK-net and F-net of the National Research Institute for Earth Science and Disaster Prevention. Generic Mapping Tools (Wessel \& Smith 1998) were used to draw the figures. This study was supported by JSPS KAKENHI Grant Numbers 14J06763, 26282104 and 25750146, and by the Ministry of Education, Culture, Sports, Science and Technology (MEXT) of Japan, under its Earthquake and Volcano Hazards Observation and Research Program.

\section{REFERENCES}

Akaike, H., 1980. Likelihood and the Bayes procedure, in Bayesian Statistics, pp. 143-166, eds Bernardo, J.M., DeGroot, M.H., Lindley, D.V. \& Smith, A.F.M., University Press.

Aki, K. \& Richards, P. G., 1980. Quantitative Seismology, Freeman, W. H., $932 \mathrm{pp}$.

Aoi, S. \& Fujiwara, H., 1999. 3D finite difference method using discontinuous grids, Bull. seism. Soc. Am., 89(4), 918-930.

Aoi, S., Kunugi, T., Nakamura, H. \& Fujiwara, H., 2011. Deployment of new strong motion seismographs of K-NET and KiK-net, in Earthquake Data in Engineering Seismology, Geotechnical, Geological, and Earthquake Engineering, vol. 14, pp. 167-186, Springer, doi:10.1007/978-94-0070152-6_12.

Asano, K., Iwata, T. \& Irikura, K., 2005. Estimation of source rupture process and strong ground motion simulation of the 2002 Denali, Alaska, earthquake, Bull. seism. Soc. Am., 95(5), 1701-1715.

Backus, G.E., 1988. Bayesian inference in geomagnetism, Geophys. J., 92(1), 125-142.

Beck, J.L., 2010. Bayesian system identification based on probability logic, Struct. Contrl. Health. Monit., 17(7), 825-847.
Burridge, R. \& Knopoff, L., 1964. Body force equivalents for seismic dislocations, Bull. seism. Soc. Am., 54(6A), 1875-1888.

Carlin, B.P. \& Louis, T.A., 2000. Bayes and Empirical Bayes Methods for Data Analysis, 2nd edn, Chapman and Hall/CRC, 440 pp.

Cohee, B. \& Beroza, G., 1994. Slip distribution of the 1992 Landers earthquake and its implications for earthquake source mechanics, Bull. seism. Soc. Am., 84(3), 692-712.

Cotton, F. \& Campillo, M., 1995. Frequency-domain inversion of strong motions: application to the 1992 Landers earthquake, J. geophys. Res., 100(B3), 3961-3975.

Dettmer, J., Benavente, R., Cummins, P.R. \& Sambridge, M., 2014. Transdimensional finite-fault inversion, Geophys. J. Int., 199(2), 735-751.

Duijndam, A.J.W., 1988. Bayesian estimation in seismic inversion. Part 1: Principles, Geophys. Prospect., 36(8), 878-898.

Duputel, Z., Agram, P.S., Simons, M., Minson, S.E. \& Beck, J.L., 2014. Accounting for prediction uncertainty when inferring subsurface fault slip, Geophys. J. Int., 197(1), 464-482.

Fukahata, Y., Nishitani, A. \& Matsu'ura, M., 2004. Geodetic data inversion using $\mathrm{ABIC}$ to estimate slip history during one earthquake cycle with viscoelastic slip-response functions, Geophys. J. Int., 156(1), $140-153$. 
Fukuda, J. \& Johnson, K.M., 2008. A fully Bayesian inversion for spatial distribution of fault slip with objective smoothing, Bull. seism. Soc. Am., 98(3), 1128-1146.

Fukuda, J. \& Johnson, K.M., 2010. Mixed linear-non-linear inversion of crustal deformation data: Bayesian inference of model, weighting and regularization parameters, Geophys. J. Int., 181(3), 14411458.

Fukuyama, E. \& Irikura, K., 1986. Rupture process of the 1983 Japan Sea (Akita-Oki) earthquake using a waveform inversion method, Bull. seism. Soc. Am., 76(6), 1623-1649.

Gelman, A., Roberts, G.O. \& Gilks, W.R., 1996. Efficient Metropolis jumping rules, in Bayesian Statistics, vol. 5, pp. 599-608, eds Bernado, J.M. et al., Oxford University Press.

George, C., 1985. An introduction to empirical Bayes data analysis, Am. Stat., 39(2), 83-87.

Geweke, J., 1992. Evaluating the accuracy of sampling-based approaches to the calculation of posterior moments, in Bayesian Statistics, vol. 4, pp. 169-193, eds Bernardo, J.M., Berger, J., Dawid, A.P. \& Smith, A.F.M., Oxford University Press.

Graves, R.W., 1996. Simulating seismic wave propagation in 3D elastic media using staggered-grid finite differences, Bull. seism. Soc. Am., 86(4), 1091-1106.

Hartzell, S.H. \& Heaton, T., 1983. Inversion of strong ground motion and teleseismic waveform data for the fault rupture history of the 1979 Imperial Valley, California, earthquake, Bull. seism. Soc. Am., 73(6), 15531583.

Hastings, W.K., 1970. Monte Carlo sampling methods using Markov chains and their applications, Biometrika, 57(1), 97-109.

Ide, S., Takeo, M. \& Yoshida, Y., 1996. Source process of the 1995 Kobe earthquake: determination of spatio-temporal slip distribution by Bayesian modeling, Bull. seism. Soc. Am., 86(3), 547-566.

Ji, C., Wald, D. \& Helmberger, D.V., 2002. Source description of the 1999 Hector Mine, California Earthquake. Part I: wavelet domain inversion theory and resolution analysis, Bull. seism. Soc. Am., 92(4), 1192-1207.

Koketsu, K., Miyake, H. \& Suzuki, H., 2012. Japan Integrated Velocity Structure Model Version 1, Paper No. 1773 presented at 15th World Conference on Earthquake Engineering, Int. Assoc. for Earthquake Eng., Lisbon, 24-28 September.

Kubo, H. \& Kakehi, Y., 2013. Source process of the 2011 Tohoku earthquake estimated from the joint inversion of teleseismic body waves and geodetic data including seafloor observation data: source model with enhanced reliability by using objectively determined inversion settings, Bull. seism. Soc. Am., 103(2B), 1195-1220.

Kubo, H., Asano, K. \& Iwata, T., 2013. Source-rupture process of the 2011 Ibaraki-oki, Japan, earthquake $\left(M_{\mathrm{W}}\right.$ 7.9) estimated from the joint inversion of strong-motion and GPS data: relationship with seamount and Philippine Sea Plate, Geophys. Res. Lett., 40(12), 30033007.

Maruyama, T., 1963. On the force equivalents of dynamic elastic dislocations with reference to the earthquake mechanism, Bull. Earthq. Res. Inst. Univ. Tokyo, 41, 467-486.

Metropolis, N., Rosenbluth, A.W., Teller, A.H. \& Teller, E., 1953. Equation of state calculations by fast computing machines, J. Chem. Phys., 21(6), $1087-1092$.

Minson, S.E., Simons, M. \& Beck, J., 2013. Bayesian inversion for finite fault earthquake source models. I-Theory and algorithm, Geophys. $J$. Int., 194(3), 1701-1726.
Minson, S.E. et al., 2014. Bayesian inversion for finite fault earthquake source models. II-The 2011 great Tohoku-oki, Japan earthquake, Geophys. J. Int., 198(2), 922-940.

Monelli, D. \& Mai, P., 2008. Bayesian inference of kinematic earthquake rupture parameters through fitting of strong motion data, Geophys. J. Int., 173(1), 1, 220-232.

Monelli, D., Mai, P.M., Jónsson, S. \& Giardini, D., 2009. Bayesian imaging of the 2000 Western Tottori (Japan) earthquake through fitting of strong motion and GPS data, Geophys. J. Int., 176(1), 135-150.

Murotani, S., Miyake, H. \& Koketsu, K., 2008. Scaling of characterized slip models for plate-boundary earthquakes, Earth Planets Space, 60, 987-991.

Nishimura, T., Hirasawa, T., Miyazaki, S., Sagiya, T., Tada, T., Miura, S. \& Tanaka, K., 2004. Temporal change of interplate coupling in northeastern Japan during 1995-2002 estimated from continuous GPS observations, Geophys. J. Int., 157(2), 901-916.

Okada, Y., Kasahara, K., Hori, S., Obara, K., Sekiguchi, S., Fujiwara, H. \& Yamamoto, A., 2004. Recent progress of seismic observation networks in Japan: Hi-net, F-net, K-NET and KiK-net, Earth Planets Space, 56, $\mathrm{xv}-\mathrm{xxviii}$.

Olson, A.H. \& Apsel, R.J., 1982. Finite faults and inverse theory with applications to the 1979 Imperial Valley earthquake, Bull. seism. Soc. Am., 72(6A), 1969-2001.

Razafindrakoto, H.N.T. \& Mai, P.M., 2014. Uncertainty in earthquake source imaging due to variations in source time function and earth structure, Bull. seism. Soc. Am., 104(2), 855-874.

Sagiya, T., 1999. Interplate coupling in the Tokai District, Central Japan, deduced from continuous GPS data, Geophys. Res. Lett., 26(15), 23152318.

Sekiguchi, H., Irikura, K. \& Iwata, T., 2000. Fault geometry in the rupture termination of the 1995 Hyogo-ken Nanbu earthquake, Bull. seism. Soc. Am., 90(1), 117-133.

Shiono, K., 2007. Introduction to numerical calculation for geological data processing, Geoinformatics, 18(4), 195-218.

Tanaka, M., Asano, K., Iwata, T. \& Kubo, H., 2014. Source rupture process of the 2011 Fukushima-ken Hamadori earthquake: how did the two subparallel faults rupture?, Earth Planets Space, 66, 101, doi:10.1186/18805981-66-101.

Tarantola, A., 2005. Inverse Problem Theory and Methods for Model Parameter Estimation, SIAM, 352 pp.

Uchida, N., Nakajima, J., Hasegawa, A. \& Matsuzawa, T., 2009. What controls interplate coupling?: evidence for abrupt change in coupling across a border between two overlying plates in the NE Japan subduction zone, Earth Planet. Sci. Lett., 283, 111-121.

Wang, L., Zöller, G. \& Hainzl, S., 2015. Joint determination of slip and stress drop in a Bayesian inversion approach: a case study for the 2010 M8.8 Maule earthquake, Pure appl. Geophys., 172, 375-388.

Wessel, P. \& Smith, W.H.F., 1998. New, improved version of Generic Mapping Tools released, EOS, Trans. Am. geophys. Un., 79, 579.

Yabuki, T. \& Matsu'ura, M., 1992. Geodetic data inversion using a Bayesian information criterion for spatial distribution of fault slip, Geophys. J. Int., 109(2), 363-375.

Yagi, Y. \& Fukahata, Y., 2011. Introduction of uncertainty of Green's function into waveform inversion for seismic source processes, Geophys. $J$. Int., 186(2), 711-720.

Yoshida, S., 1989. Waveform inversion using ABIC for the rupture process of the 1983 Hindu Kush earthquake, Phys. Earth planet. Inter., 56, 389-405.

\section{SUPPORTING INFORMATION}

Additional Supporting Information may be found in the online version of this paper:

Figure S1. Observed waveforms used in the synthetic test of Fig. 14. Black and red waveforms were calculated from the input source model in Fig. 14(a) with no noise and 20 per cent Gaussian noise, respectively. 
Figure S2. Final slip distribution of input source models (upper) and estimated source models (lower) in synthetic tests where the input models have two patches with the same slip amount $(3 \mathrm{~m})$. Synthetic waveforms of the synthetic tests have a 20 per cent Gaussian noise. The inversion settings in the synthetic tests were the same as those used in the analysis of actual data.

Figure S3. Final slip distribution of input source models (upper) and estimated source models (lower) in synthetic test where the input models have two patches with different slip amounts $(2.5 \mathrm{~m}$ and $3.5 \mathrm{~m})$. Synthetic waveforms of the synthetic tests have a 20 per cent Gaussian noise. The inversion settings in the synthetic tests were the same as those used in the analysis of actual data. (http://gji.oxfordjournals.org/lookup/suppl/doi:10.1093/gji/ggv540/-/DC1).

Please note: Oxford University Press is not responsible for the content or functionality of any supporting materials supplied by the authors. Any queries (other than missing material) should be directed to the corresponding author for the paper. 